\title{
Action Selection for Single-Camera SLAM
}

\author{
Teresa A. Vidal-Calleja, Alberto Sanfeliu, and Juan Andrade-Cetto, Member, IEEE
}

\begin{abstract}
A method for evaluating, at video rate, the quality of actions for a single camera while mapping unknown indoor environments is presented. The strategy maximizes mutual information between measurements and states to help the camera avoid making ill-conditioned measurements that are appropriate to lack of depth in monocular vision systems. Our system prompts a user with the appropriate motion commands during 6-DOF visual simultaneous localization and mapping with a handheld camera. Additionally, the system has been ported to a mobile robotic platform, thus closing the control-estimation loop. To show the viability of the approach, simulations and experiments are presented for the unconstrained motion of a handheld camera and for the motion of a mobile robot with nonholonomic constraints. When combined with a path planner, the technique safely drives to a marked goal while, at the same time, producing an optimal estimated map.
\end{abstract}

Index Terms-Action selection, active vision, bearing-only simultaneous localization and mapping (SLAM), mutual information, path planning.

\section{INTRODUCTION}

A UTONOMOUS vehicles must be able to automatically determine their control commands to achieve a specified task. Commonly, it is assumed that the vehicle has complete and exact knowledge of its environment; of course, this assumption is not always realistic. If uncertainty in the prior knowledge is small, it is reasonable to anticipate all possible contingencies and to generate sensor-based motion plans that can deal with them. On the other hand, if the autonomous vehicle has no prior knowledge of its environment, it is necessary to first learn about it.

Mobile robots operate in environments that are either partially or completely unknown. Often, the environment is changing with time in an unknown manner; hence, sensors that can enable the vehicle to navigate in these environments are well motivated.

Vision sensors offer a wide field of view, plus millisecond sampling rates, and thus can easily be used for control. Cameras can be cheaper when compared to other sensors, such as laser

Manuscript received August 27, 2009; accepted January 10, 2010. Date of publication March 25, 2010; date of current version November 17, 2010. This work was supported by the Spanish Ministry of Science and Innovation under Projects UbROB (DPI 2007-61452), PAU (DPI2008-06022), and MIPRCV (Consolider-Ingenio 2010), and the EU URUS Project IST-FP6STREP-045062. This paper was recommended by Associate Editor H. Zhang.

T. A. Vidal-Calleja is with the Institut de Robòtica i Informàtica Industrial, CSIC-UPC, 08028 Barcelona, Spain, on leave at the Australian Centre for Field Robotics, University of Sydney, Sydney, NSW 2006, Australia.

A. Sanfeliu and J. Andrade-Cetto are with the Institut de Robòtica i Informàtica Industrial, CSIC-UPC, 08028 Barcelona, Spain.

Color versions of one or more of the figures in this paper are available online at http://ieeexplore.ieee.org.

Digital Object Identifier 10.1109/TSMCB.2010.2043528 range scanners. However, some disadvantages of vision include lack of depth information, image occlusion, low resolution, and the requirement for extensive data interpretation, i.e., recognition.

Real-time performance imposes severe restrictions on the volume of computation that can take place in a time step. In order to get a fully autonomous vehicle working in a partially unknown environment, image processing, decision making, full estimation of vehicle location, and map updating should be done at video rates, i.e., at 16 or $33 \mathrm{~ms} / \mathrm{step}$.

This paper is about the guidance of an autonomous vehicle using only a single camera. We are interested in the real-time estimation and control of a single camera's motion, moving in 3-D within normal human environments and on nonflat terrains, mapping visual features. The presented technique builds a feature map and localizes the sensor in this map by computing the appropriate control actions in order to improve overall system estimation.

Three experimental settings are discussed. The first one is an online implementation for a single-camera simultaneous localization and mapping (SLAM) system that extends the work of Davison [1], adding control to his otherwise passive monocular system. Given the real-time characteristics of the visual SLAM system used, fast and efficient action evaluation is of utmost importance. Fortunately enough, the elements needed to validate the quality of actions with respect to entropy reduction are readily available from the SLAM priors, and by making enough implementation adaptations, we are able to evaluate in real time the value of a reasonably sized action space in order to give a human approximate low-frequency easy-to-understand motion commands: 'move forward', 'turn left', stop', and so on. An earlier version of this online implementation is presented in [2]. The technique is extended in this paper with a novel more efficient method to compute the information gain in constant time.

The second setting discussed in this paper depicts a wideangle camera mounted on a mobile robot navigating in uneven terrain. In this case, the expected information gain is evaluated by propagating a particular action using a constrained motion model. This model considers not only the nonholonomic constraints of the vehicle but also the slope of the terrain. As in the previous case, the action space is also discretized but, this time, within the range of possible translational and angular impulse accelerations of the mobile robot. An early version of the technique was presented in [3]. In contrast to our contributions in [2] and [3], this paper contains simulations averaging the results of multiple Monte Carlo simulations for the aforementioned two systems, as well as a consistency analysis for the handheld camera case. The results of these extended simulation results provide stronger support for the effectiveness of the approach. 
Finally, a third case that combines the action selection strategy with a local navigation technique, allowing for both entropy reduction and obstacle avoidance at the same time, is presented. The method proposes a new motion strategy that combines a local planner with our information theoretic metric. Extended simulation results are also reported for this latter case.

The remainder of this paper is ordered as follows: First, we present a summary of related work. Then, in Section III, a brief introduction is given on the extended-Kalman-filterbased single-camera SLAM estimation. The information gain metric that will be used to test the action space in the search for a locally optimal action is also detailed. In Section IV, we present an overview of the motion models for the freemoving handheld camera and the constrained motion of a wheeled mobile robot. In the same section, we also present the local navigation strategy chosen. Section V is devoted to the general perception model. Section VI discusses in detail three experimental settings, including both simulations and experimental results. First, the handheld camera performing SLAM at video rate with generic 6-DOF motion is shown, where a graphical user interface (GUI) feeds back motion commands to the user. The section shows the advantages of using the proposed action selection strategy when compared with random exploratory sequences. Second, the case of a camera mounted on a vehicle moving on uneven 3-D terrain is given, in which a low-level feedback controller is used to follow the actions chosen by the mutual information-based exploration strategy. Finally, simulation results are presented for the application of the local navigation technique, together with our action selection strategy. Concluding remarks are given in Section VII.

\section{RELATED WORK}

Considerable research has focused on the use of vision systems for guiding autonomous vehicles without mapping. As exemplars, Kim et al. [4] proposed a tracking system based on monocular visual feedback using consecutive image frames and an object database. This was achieved with the use of an EKF. Das et al. [5] also used an EKF and feedback from a monocular omnidirectional camera to enable wall-following, followthe-leader, and position regulation tasks. In [6], a method to stabilize a wheeled mobile robot to a target pose based on the discrepancies between a target view of the landmarks in the workspace and the robot's current view is presented. The method combines the nonlinear control theory with research derived from hypotheses on insect navigation. In [7], a bionic vision system is used to guide a humanoid robot combining a mechanical platform for pitching and rolling with stereo vision to obtain the position of the objects in the scene.

In a different context, Song and Huang [8] used spatiotemporal apparent velocities obtained from an optical flow of successive images of an object to estimate depth and time-tocontact to develop a monocular vision guided robot. In [9], Wang et al. exploited rigid body transformations to develop a visually servoed mobile robot (the regulation problem was not solved due to restrictions on the reference trajectory) that adapted for the constant unknown height of an object moving on a plane. In a mapping context, without guidance, optical flow, kinematics, and depth information are used to estimate the object's motion in [10].

One of the first approaches that took into account the effect of actions during mapping with visual sensing used feature correspondences from stereo image pairs [11]. The computational burden for the accurate detection and matching of image pairs motivated the use of active visual sensing for landmark selection in sparse feature maps.

Action selection is of paramount importance during bearingonly SLAM. The reason is that single-bearing observations produce ill-posed depth estimates, whereas incremental-bearing observations can be integrated with the appropriate selection of camera motion for triangulation. Our thesis is that the camera should be driven to a location that maximizes the expected information gain between states and measurements. Exploration strategies driven by uncertainty reduction have been used in the past for the acquisition of 3-D models of objects from range data [12] and within the context of SLAM to produce maps from ultrasonic signals [13]. In the latter work, a metric is proposed to evaluate uncertainty reduction as the sum of the independent robot and landmark entropies with an exploration horizon of one step. Bourgault et al. [14] alternatively proposed a utility function for exploration that trades off the cost of navigation with the potential vehicle localization uncertainty reduction (measured as entropy) and the information gained over an occupancy grid. That work required two different representations of the environment: 1) an occupancy grid and 2) a map of individual features. Their work suggested results over a simulated SLAM setting for a 2-D mobile platform with range sensing. In contrast to these approaches, which independently consider the reduction of vehicle and map entropies, we tackle the issue of joint robot and map entropy reduction, taking into account robot and map cross correlations. Our work uses a feature-based map representation, and we present results not only over simulated settings but also for real-time implementations.

Another technique that tackles the problem of exploration in SLAM as an entropy minimization problem makes use of Rao-Blackwellized particle filters [15]. When using particle filters for exploration, only a very narrow action space can be evaluated due to the complexity in computing the expected information gain. The main bottleneck is the generation of the expected measurements that each action sequence would produce, which is generated by a ray-casting operation in the map of each particle. In contrast, measurement predictions in a feature-based EKF implementation, such as ours, can be computed much faster, having only one map posterior per action to evaluate, instead of the many that a particle filter requires. Moreover, in [15], the cost of choosing a given action is subtracted from the expected information gain with a userselected weighting factor. In this paper, we show how the cost of performing a given action is inherently taken into account when evaluating the entropy for a set of possible priors. Higher level strategies such as planning in partially observable continuous domains via value iteration over POMDPs could also be considered [16]. Unfortunately, such long-term planning approaches are not yet viable for real-time implementations and are out of the scope of this work. 
In a work closely related to ours, Bryson et al. presented simulated results of the effect that different vehicle actions have with respect to the entropic mutual information gain [17]. The analysis is performed for a 6-DOF aerial vehicle equipped with one camera and an inertial sensor, for which landmark range, azimuth, and elevation readings are simulated, and data association is known.

In all of the previously discussed works, little to no effort has been expended on the real-time constraints of action selection during exploration. This paper instead pays special attention to ensure that the most appropriate action is chosen within the small computation time available for video-rate bearing-only SLAM systems.

\section{ACTION SELECTION}

This section presents the strategy of choosing the appropriate motion commands for a single camera moving about an unknown environment. The aim is to move the camera in the direction that most reduces the uncertainty in the entire SLAM state by using the information that should be gained from future predicted landmark observations should such a move take place but taking into account the information lost as a result of moving with uncertainty.

Consider the classical feature-based SLAM framework [18], in which the state vector $\mathrm{x}$ contains the camera pose and the map of features $\mathbf{x}=\left(\mathbf{x}_{v}, \mathbf{y}\right)$. An EKF propagates the camera pose and velocity estimates, as well as the map estimate. The state prior $\mathbf{x}_{k+1 \mid k}$ is predicted with the motion models described in Section IV, which slightly vary, depending on the platform setup. For instance, a handheld camera freely moving is tracked with a constant velocity model, whereas a robot moving in uneven terrain is restricted to be in contact with the ground surface at all times, and its motion is limited by its nonholonomic constraints.

In both cases, linear approximations for uncertainty propagation are computed with $\boldsymbol{\Sigma}_{k+1 \mid k}=\mathbf{F} \boldsymbol{\Sigma}_{k \mid k} \mathbf{F}^{\top}+\boldsymbol{\Sigma}_{u}$, in which $\mathbf{F}$ is the appropriate motion model Jacobian, which is computed as the partial derivative with respect to the state $\mathbf{x}$ of the motion models for the free-moving camera and for the constrained robot motion, and $\boldsymbol{\Sigma}_{u}$ is the input motion covariance term, which is adequately tuned for each of the systems.

The subscripts $k \mid k$ and $k+1 \mid k$ denote the posterior at time $k$ and the prior (before integrating measurements) at time $k+1$, respectively. State updates follow the conventional Kalman recursion $\mathbf{x}_{k+1 \mid k+1}=\mathbf{x}_{k+1 \mid k}+\mathbf{K}\left(\mathbf{z}_{i}-\mathbf{z}_{i, k+1 \mid k}\right)$, with $\mathbf{z}_{i, k+1 \mid k}$, which is the $i$ th measurement prediction, and for which the Kalman gain, error covariance matrix, and covariance update are computed with

$$
\begin{aligned}
\mathbf{K} & =\boldsymbol{\Sigma}_{k+1 \mid k} \mathbf{H}^{\top} \mathbf{S}^{-1} \\
\mathbf{S} & =\mathbf{H} \boldsymbol{\Sigma}_{k+1 \mid k} \mathbf{H}^{\top}+\boldsymbol{\Sigma}_{z} \\
\boldsymbol{\Sigma}_{k+1 \mid k+1} & =(\mathbf{I}-\mathbf{K H}) \boldsymbol{\Sigma}_{k+1 \mid k}
\end{aligned}
$$

with $\mathbf{H}$, which is the Jacobian of the measurement model with respect to the state. Measurement uncertainty is assumed to be zero-mean Gaussian with covariance $\boldsymbol{\Sigma}_{z}$.

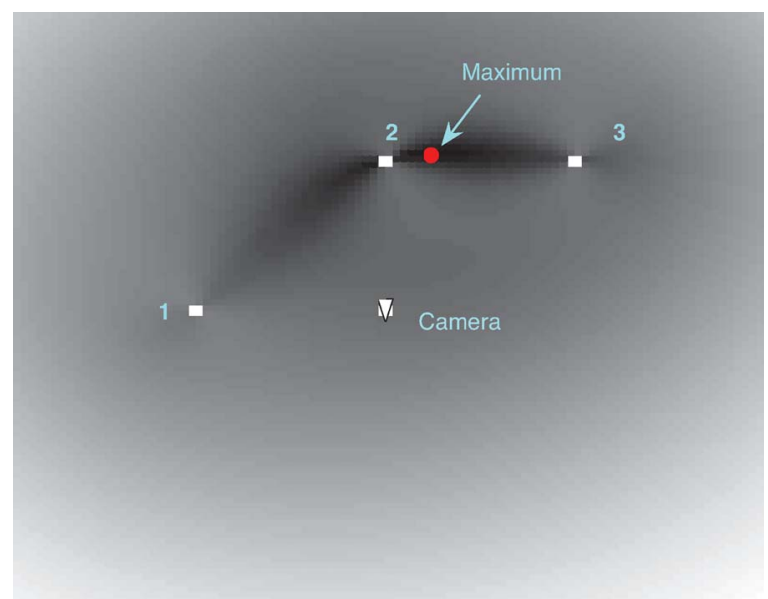

Fig. 1. Maximization of mutual information for the evaluation of motion commands. A bearing sensor is located at the center of the plot, and a decision on where to move must be taken as a function of the pose and landmark states, and the expected measurements. Three landmarks are located to its left, front, and right-front. Moving to the location in between landmarks 2 and 3 maximizes the mutual information between the SLAM prior and the measurements.

We adopt entropy as a measure of dispersion, i.e., as a measure of how much randomness there is in our state estimate. Entropy is defined as $H(X)=-\sum_{\mathbf{x}} p(\mathbf{x}) \log p(\mathbf{x})$, which, for our case where $p(\mathbf{x})$ is an $n$-variate Gaussian distribution, has the form $H(X)=1 / 2 \log \left((2 \pi e)^{n}|\boldsymbol{\Sigma}|\right)$.

Being able to know the changes in entropy naturally leads to the important question "What action provides the best reduction of entropy?" or, equivalently, "What action gives us the most informative observation?" The question may be answered through the idea of mutual information.

We want to choose the action that maximizes the mutual information between the state $\mathrm{x}$ and the possible measurements occurring at that location $\mathbf{z}$. The mutual information is defined as the relative entropy between the joint distribution $p(\mathbf{x}, \mathbf{z})$ and the marginals $p(\mathbf{x})$ and $p(\mathbf{z})$, i.e.,

$$
\begin{aligned}
I(X Z) & =\sum_{\mathbf{x} \in X, \mathbf{z} \in Z} p(\mathbf{x}, \mathbf{z}) \log \frac{p(\mathbf{x}, \mathbf{z})}{p(\mathbf{x}) p(\mathbf{z})} \\
& =H(X)+H(Z)-H(X, Z) \\
& =H(X)-H(X \mid Z)
\end{aligned}
$$

which, for our Gaussian multivariate case, evaluates to

$$
I(X Z)=\frac{1}{2} \log \frac{\left|\boldsymbol{\Sigma}_{k+1 \mid k}\right|}{\left|\boldsymbol{\Sigma}_{k+1 \mid k+1}\right|} .
$$

In choosing a maximally mutually informative motion command, we are maximizing the difference between prior and posterior entropies [19]. That is, we choose the motion command that most reduces the uncertainty of $x$ due to the knowledge of $\mathrm{z}$ as a result of a particular action. Fig. 1 shows the directions maximizing the mutual information for a simple 2-DOF camera and three landmarks. The cost of actions is implicitly taken into account as loss of information when evaluating the priors in (2). This is possible, because the plant noise model depends on the value of the motion command. In the figure, the entropy 


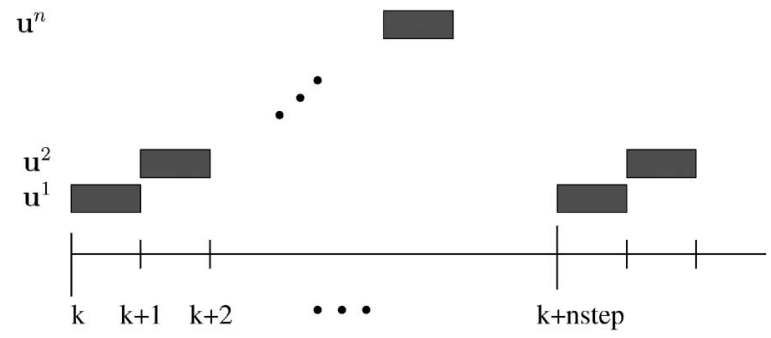

Fig. 2. Action evaluation in time intervals. Actions $U=\left\{\mathbf{u}^{1}, \mathbf{u}^{2}, \ldots, \mathbf{u}^{n}\right\}$ are evaluated at different time intervals but compared at the same instant in time.

reduction as a result of the three landmark-bearing measurements is plotted in grayscale. The optimal position, for which the relative entropy is maximized, is marked by the red dot in the plot. Note also that, for large-valued actions, the compromise between greater loss of information during the prediction step and the information recovery during the update step produces less informative overall entropy reduction. This happens near the boundaries of the action space shown.

This action selection mechanism picks the optimal choice in one-step lookahead. Getting the optimal sequence of actions for a larger time horizon turns out to be prohibitive for a realtime application. The problem turns into a partially observable Markov decision process, in which computing the value function would require the integration of all possible states and measurements. Even for the one-step lookahead time horizon, the evaluation of a decently sized action space requires some considerations. The computation of determinants in (2) only allows for the estimates of the outcome of all possible actions from the set $U$ to be computed for neighboring points in time. However, the comparison against each other occurs at the same point in time. Fig. 2 illustrates the approach.

A second consideration is necessary to drive the system to explore and not to settle for homoeostasis, because the use of entropy reduction as a measure of exploration assumes that uncertainty can be reduced as landmarks are being discovered. Our solution to the problem is to first have an idea of the size of the space to be mapped and initially fill this space with uniformly distributed expected landmarks with large uncertainty. The error covariance matrix that will be used in computing the information gain measures contains the variances for such a fixed number of uncorrelated unvisited landmarks. Fig. 3 shows a representation of such covariance matrix. It is common practice to have an a priori estimation of the size to be mapped for any mobile robotic exploration mechanism. Think, for example, of probabilistic grid-based mapping. The grid size and granularity are known a priori. In our case, the expected number of landmarks to see and a very rough uniform disposition of them in the environment are our initial conditions. Several authors make such assumption either with a priori grid-based discretization of the environment [14], [20] or by adding uniformly distributed unvisited landmarks as vague priors [21], [22].

A straightforward evaluation of global entropy reduction as in (2) is computationally expensive. It requires the computation of the determinants of large matrices. In recent work [23], we show however that this measure can be computed in constant
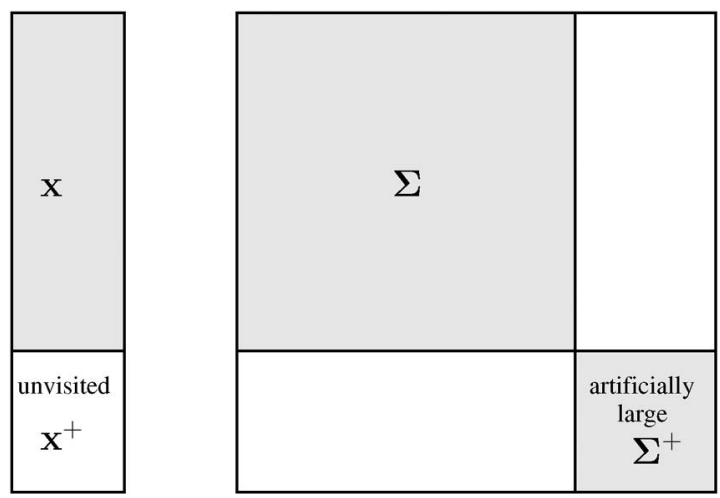

Fig. 3. A fixed number of unvisited landmark states accounting for the unexplored part of the scene is added to the state vector during the computation of entropy values. These unvisited landmarks contain artificially large and uncorrelated covariance.

time. Algebraic manipulation transforms the ratio of determinants of large matrices in (2) into a ratio of determinants of matrices with the size of the observation

$$
I=\frac{1}{2} \log \frac{\left|\boldsymbol{\Sigma}_{k+1 \mid k}\right|}{\left|\boldsymbol{\Sigma}_{k+1 \mid k+1}\right|}=\frac{1}{2} \log \frac{|\mathbf{S}|}{\left|\boldsymbol{\Sigma}_{z}\right|} .
$$

Due to the sparsity of the measurement Jacobian $\mathbf{H}$, the computation of the preceding equation turns out to be independent of the map size and can be computed in constant time, depending only on the range of the sensor. To be more precise, the computation takes time cubic on the number of features seen at each iteration. The result is particularly relevant for not only exploration strategies such as the one reported in this paper but also SLAM systems, in general, that use information content at each step for other purposes, such as active loop closure assertion or heuristics that maintain the state size limited.

\section{Motion Models And Local Navigation}

The three experimental settings presented in this work use the preceding considerations with slight differences.

\section{A. Unconstrained Constant Velocity Motion Model}

The first experimental setting focuses on action selection for video-rate SLAM with a single camera rapidly moving with 6-DOF in 3-D within normal human environments and with minimal prior information about motion dynamics. Insisting on video-rate performance using modest hardware imposes severe restrictions on the volume of computation that can take place in each 16-ms time step. Re-estimation must take place of course, but making strictly optimal camera movements would require, in addition, the computation of model Jacobians for the entire action space [24]. Such a computation remains infeasible for our 6-DOF nonlinear system and measurement models. Human actions are 1) approximate, 2) low frequency, and 3) delayed. Therefore, we give a small set of choices at 1 -s intervals. To give a human time to react, the action is selected every second, so in Fig. 2, nstep is equal to the frames per second. The camera motion predictions are computed with a smooth unconstrained constant velocity motion model presented as follows: 
It is assumed that the camera could be attached to any mobile platform - in this case, a human hand-and is free to move in any direction in $\mathbb{R}^{3} \times S O(3)$. The system is driven by zero-mean normally distributed accelerations. The Gaussian acceleration assumption means that large impulsive changes of direction are unlikely. The camera motion prediction model is

$$
\mathbf{x}_{v, k+1 \mid k}=\left[\begin{array}{c}
\mathbf{p}_{k+1 \mid k} \\
\mathbf{q}_{k+1 \mid k} \\
\mathbf{v}_{k+1 \mid k} \\
\boldsymbol{\omega}_{k+1 \mid k}
\end{array}\right]=\left[\begin{array}{c}
\mathbf{p}_{k \mid k}+\left(\mathbf{v}_{k \mid k}+\mathbf{a}_{k} \Delta t\right) \Delta t \\
\mathcal{Q}_{k \mid k} \\
\mathbf{v}_{k \mid k}+\mathbf{a}_{k} \Delta t \\
\boldsymbol{\omega}_{k \mid k}+\boldsymbol{\alpha}_{k} \Delta t
\end{array}\right]
$$

with $\mathbf{p}=(x, y, z)^{\top}$ and $\mathbf{q}=\left(q_{0}, q_{1}, q_{2}, q_{3}\right)^{\top}$ denoting the camera pose (three states for position and four for orientation using a unit norm quaternion representation), and $\mathbf{v}=\left(v_{x}, v_{y}, v_{z}\right)^{\top}$ and $\boldsymbol{\omega}=\left(\omega_{x}, \omega_{y}, \omega_{z}\right)^{\top}$ denoting the linear and angular velocities, respectively. The input to the system is the acceleration vector $\mathbf{u}=\left(\mathbf{a}^{\top}, \boldsymbol{\alpha}^{\top}\right)^{\top}=\left(a_{x}, a_{y}, a_{z}, \alpha_{x}, \alpha_{y}, \alpha_{z}\right)^{\top}$.

The model $\mathcal{Q}$ for the prediction of change in orientation is inspired by [25] and is

$$
\mathcal{Q}=\cos \left(\frac{\Delta t\|\boldsymbol{\Omega}\|}{2}\right) \mathbf{I}+\frac{2}{\|\boldsymbol{\Omega}\|} \sin \left(\frac{\Delta t\|\boldsymbol{\Omega}\|}{2}\right) \mathbf{M}
$$

where $\mathbf{M}$ is the skew-symmetric matrix form of the angular velocity

$$
\mathbf{M}=\left[\begin{array}{cccc}
0 & -\omega_{x} & -\omega_{y} & -\omega_{z} \\
\omega_{x} & 0 & -\omega_{z} & \omega_{y} \\
\omega_{y} & \omega_{z} & 0 & -\omega_{x} \\
\omega_{z} & -\omega_{y} & \omega_{x} & 0
\end{array}\right]
$$

The redundancy in the quaternion representation is accounted with a $\|\mathbf{q}\|=1$ normalization at each update, accompanied by the corresponding Jacobian modification as in [1]. Note also that this model is decoupled in terms of linear and angular velocities. Therefore, camera rotations do not affect the translation.

\section{B. Constrained 3-D Motion Model}

In the second experiment, the case of the camera mounted on a vehicle moving in uneven terrain is considered. We have opted for a strategy that chooses actions in terms of impulse accelerations. The expected information gain is evaluated by propagating actions with a constrained motion model, with the advantage that this model considers not only the nonholonomic constraints of the vehicle but also the slope of the terrain. The vehicle receives a new control command when all the actions have completely been evaluated, i.e., nstep in Fig. 2 is equal to the size of $U$ plus one.

In order to do the prediction step of the EKF, computing the position using the 2-D vehicle model or even the odometry information would be inappropriate if we want 3-D information. For this reason, the model considers not only uneven surfaces but also the kinematic constraints of the differential steer vehicle and the camera position with respect to the rotation axis of the vehicle.
In this case, the vehicle is controlled by linear and angular velocities $\mathbf{u}=\left(v_{r}, \omega_{r}\right)^{\top}$, which are tangent to the terrain surface. Vehicle surface contact is considered at all times. Substituting the previous motion prediction model with a constrained model for the continuous transition of the camera optic center, we get

$$
\left[\begin{array}{l}
\mathbf{p}_{k+1} \\
\boldsymbol{\theta}_{k+1}
\end{array}\right]=\left[\begin{array}{l}
\mathbf{p}_{k} \\
\boldsymbol{\theta}_{k}
\end{array}\right]+\left[\begin{array}{l}
\mathbf{F}_{v} \\
\mathbf{F}_{\omega}
\end{array}\right] \mathbf{u}_{k} \Delta t
$$

where

$$
\begin{aligned}
\mathbf{F}_{v} & =\left[\begin{array}{cc}
-\sin \phi \sin \psi-\cos \phi \cos \psi \sin \theta & -l \cos \psi \cos \theta \cos \phi \\
\cos \phi \sin \psi-\sin \phi \cos \psi \sin \theta & -\cos \psi \cos \theta \sin \phi \\
\cos \psi \cos \theta & -l \cos \psi \sin \theta
\end{array}\right] \\
\mathbf{F}_{\omega} & =\left[\begin{array}{cc}
0 & \sin \psi \tan \theta \\
0 & \cos \psi \\
0 & \frac{\sin \psi}{\cos \theta}
\end{array}\right]
\end{aligned}
$$

$\boldsymbol{\theta}=[\psi, \theta, \phi]^{\top}$ is a yaw-pitch-roll Euler angle representation of $\mathbf{q}$, and $l$ is the distance between the axle center of the mobile robot and the camera optic center.

\section{Vehicle Guided With APF}

The third experiment combines the uncertainty reduction strategy with a local planner in order to guide the vehicle from an initial to a final position without colliding with obstacles. The control law for the vehicle is based on an artificial potential field (APF). In using the potential field method, the vehicle follows the direction of a resultant force that combines attractive and repulsive forces to guide the vehicle through obstacles to reach a goal. The strategy considers optimal actions as the maximum reduction of entropy added to the direction of the potential field. In this way, the vehicle is able to reduce uncertainty in the map and in its pose while being guided to its final destination without colliding with the obstacles of the map.

The APF method has extensively been studied in the obstacle avoidance problem for autonomous mobile robots [26]-[28] in convex terrains. Control for the vehicle using this approach is presented as follows:

Consider the position of the vehicle in a workspace $\mathbf{p}$ [refer to (7)], the position of the goal g, and the position of an obstacle, e.g., y. An artificial potential function applied to the vehicle at point $\mathbf{p}$ has the form

$$
U=U_{a}(\mathbf{p}, \mathbf{g})+U_{r}(\mathbf{p}, \mathbf{y})
$$

where $U_{a}(\mathbf{p}, \mathbf{g})$ is the attractive potential induced by the goal, and $U_{r}(\mathbf{p}, \mathbf{y})$ is the repulsive potential induced by the obstacle. The resultant force is then obtained as $\mathbf{f}=\mathbf{f}_{a}+\mathbf{f}_{r}$, where

$$
\begin{aligned}
& \mathbf{f}_{a}=-\nabla U_{a}(\mathbf{p}, \mathbf{g}) \\
& \mathbf{f}_{r}=-\nabla U_{r}(\mathbf{p}, \mathbf{y}) .
\end{aligned}
$$

The relation between the output $\mathbf{p}$ and the control input is $\dot{\mathbf{p}}=\mathbf{u}$, and the task at hand is to compute the optimal velocity command $\mathbf{u}$. 
Given that the position of the camera in the $y$-axis depends only on the terrain (the altitude is not controllable), we reduce the obstacle avoidance problem to a 2-D positioning problem on the $x z$ plane. Let us consider the desired velocity values $\mathbf{u}_{d}=\left(u_{x, d}, 0, u_{z, d}\right)$ to be proportional to the normalized force generated by the potential field, i.e.,

$$
\left[\begin{array}{l}
u_{x, d} \\
u_{y, d} \\
u_{z, d}
\end{array}\right]=\frac{u_{d}}{\|\mathbf{f}\|}\left[\begin{array}{c}
f_{1} \\
0 \\
f_{2}
\end{array}\right]
$$

where $f_{1}$ and $f_{2}$ are the components of the total force $\mathbf{f}$ in the direction of the $x$ - and $z$-axis, respectively, and $u_{d}$ is the desired scalar velocity. Under these conditions, it is possible to propose the feedback control law

$$
\left[\begin{array}{c}
v_{r} \\
\omega_{r}
\end{array}\right]=\frac{v_{d}}{\|\mathbf{f}\|} \mathbf{F}_{v}^{+}\left[\begin{array}{c}
f_{1} \\
0 \\
f_{2}
\end{array}\right] .
$$

where $\mathbf{F}_{v}^{+}=\left(\mathbf{F}_{v}^{\top} \mathbf{F}_{v}\right)^{-1} \mathbf{F}_{v}^{\top}$ is the left pseudoinverse of $\mathbf{F}_{v}$.

The attractive potential is given by

$$
U_{a}=\frac{1}{2} \xi\left(\left(z-g_{z}\right)^{2}+\left(x-g_{x}\right)^{2}\right)
$$

where $\mathbf{g}=\left(g_{x}, y_{k \mid k}, g_{z}\right)$ is the goal.

On the other hand, the repulsive potential, which is inside of the region of influence, is given by

$$
U_{r}=\frac{\eta}{2}\left(\frac{1}{\left(z-y_{z}^{i}\right)^{2}+\left(x-y_{x}^{i}\right)^{2}}-\frac{1}{\rho_{0}^{2}}\right)^{2}
$$

where $y_{x}^{i}$ and $y_{z}^{i}$ are the 2-D components of the $i$ th obstacle or map feature position vector.

The total repulsive force is given by

$$
\mathbf{f}_{r}=\sum_{i \in J} \mathbf{f}_{r}^{i}
$$

with $J=\left\{i \mid \rho\left(\mathbf{p}, \mathbf{y}^{i}\right) \leq \rho_{0}^{2}\right\}$ and $\rho\left(\mathbf{p}, \mathbf{y}^{i}\right)=\left(z-y_{z}^{i}\right)^{2}+(x-$ $\left.y_{x}^{i}\right)^{2}$, i.e., the square distance to each obstacle or feature in the workspace.

Note that, for the first two experimental settings, i.e., the handheld camera and the vehicle moving in 3-D terrain, the use of the unvisited expected landmarks is needed to drive exploration. In the case of the APF, these unknown landmarks are no longer needed. Thus, the optimal action now is given by

$$
\mathbf{u}^{*}=\lambda \arg \min _{\mathbf{u} \in U} \mathcal{I}(\mathbf{x}, \mathbf{u}, \mathbf{z})+(1-\lambda) \min U\left(\mathbf{x}_{k \mid k}\right)
$$

where $\lambda$ is the weight factor.

\section{Measurement Model}

In this paper, we are interested in mapping the 3-D coordinates of salient point features from images and need to do so at video rate. Environments with a relatively small number of features are considered (50 or less). The strategy is aimed at local action selection and can be used in any submapping or hierarchical mapping approach at the level of each local map. Thus,
TABLE I

PARAmeters For the Simulation OF HANDhEld CAMERA SLAM

\begin{tabular}{|c|c|}
\hline Initial pose & {$[0 \mathrm{~m}, 0 \mathrm{~m},-1.5 \mathrm{~m}, 0 \mathrm{rad}, 0 \mathrm{rad}, 0 \mathrm{rad}]$} \\
\hline Initial pose & {$[0.32 \mathrm{~m}, 0.22 \mathrm{~m}, 0.32 \mathrm{~m}$,} \\
standard deviation & $0 \mathrm{rad}, 0 \mathrm{rad}, 0 \mathrm{rad}]$ \\
\hline Initial & {$[0 \mathrm{~m} / \mathrm{s}, 0 \mathrm{~m} / \mathrm{s},-0.1 \mathrm{~m} / \mathrm{s}$,} \\
velocity & $0 \mathrm{rad} / \mathrm{s}, 0 \mathrm{rad} / \mathrm{s}, 0.01 \mathrm{rad} / \mathrm{s}]$ \\
\hline Model noise & $\sigma_{a}^{2}=0.15 \mathrm{~m} / \mathrm{s}^{2}, \sigma_{\alpha}^{2}=0.15 \mathrm{rad} / \mathrm{s}^{2}$ \\
\hline
\end{tabular}

the limit of 50 landmarks per local map is not a constraint to the action selection mechanism. The visual SLAM implementation used is based on the Shi-Tomasi saliency operator, matching correspondences in subsequent frames using a normalized sumof-squared differences, and a particle filter along the ray for initialization [11], [29]. Different implementations using, for instance, SIFT features and inverse depth initialization can be found in the literature and beyond the scope of this work.

Image projection is modeled using a full-perspective wideangle camera model. The position of a 3-D scene point $\mathbf{y}_{i}$ is transformed into the camera frame as $\mathbf{y}_{i}^{c}=\left[x^{c}, y^{c}, z^{c}\right]^{\top}=$ $\mathbf{R}^{\top}\left(\mathbf{y}_{i}-\mathbf{p}\right)$, with $\mathbf{R}$ being the rotation matrix equivalent to $\mathbf{q}$. The point's projection onto the image plane is

$$
\mathbf{h}_{i}=\left[\begin{array}{l}
u \\
v
\end{array}\right]=\left[\begin{array}{l}
u_{0}-u_{c} / \sqrt{d} \\
v_{0}-v_{c} / \sqrt{d}
\end{array}\right]
$$

where $u_{c}=f k_{u} x^{c} / z^{c}, v_{c}=f k_{v} y^{c} / z^{c}$, the radial distortion term is $d=1+K_{d}\left(u_{c}^{2}+v_{c}^{2}\right)$, and the intrinsic calibration of the camera (focal distance $f$, principal point $\left(u_{0}, v_{0}\right)$, pixel densities $k_{u}$ and $k_{v}$, and radial distortion parameter $K_{d}$ ) is calibrated a priori.

When an image feature is detected, its measurement must be either associated with an existing feature or added as a new feature in the map. The location of the camera, along with the locations of the already mapped features, are used to predict feature position $\mathbf{h}_{i}$ using (14), and these estimates are checked against the measurements using a nearest neighbor test. Feature search is constrained to $3 \sigma$ elliptical regions around the image estimates, as defined by the innovation covariance matrix.

\section{Simulations And ExPERIMENTAl Results}

In this section, we present simulation and experimental results for the three cases previously mentioned. The experiments show how the proposed entropy-based action selection mechanism produces improved overall localization and map estimation, compared to random motion selection for both unconstrained and constrained motion and also to the case of APF alone when driving to a predefined goal.

\section{A. Handheld Camera}

For the free-moving camera, changes in translation and orientation are kinematically decoupled. In addition, should an omnidirectional sensor be used, one would not require a strategy to direct fixation. Following this reasoning, our action selection metric is only used to decide upon maximally 


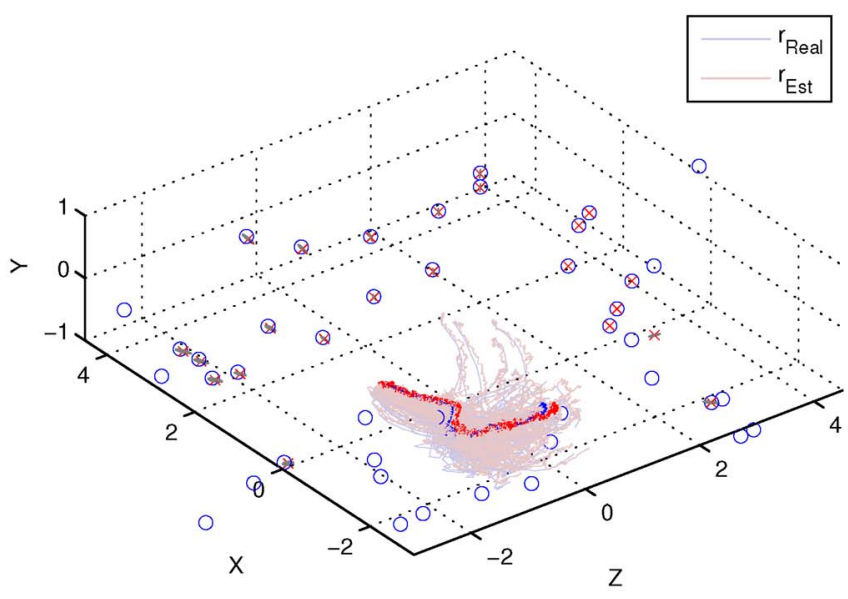

(a)

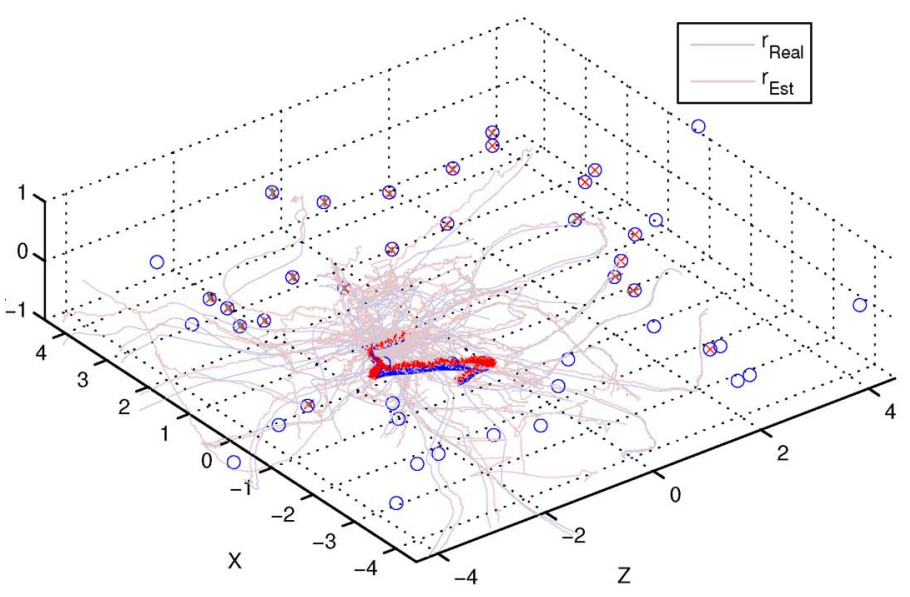

(c)

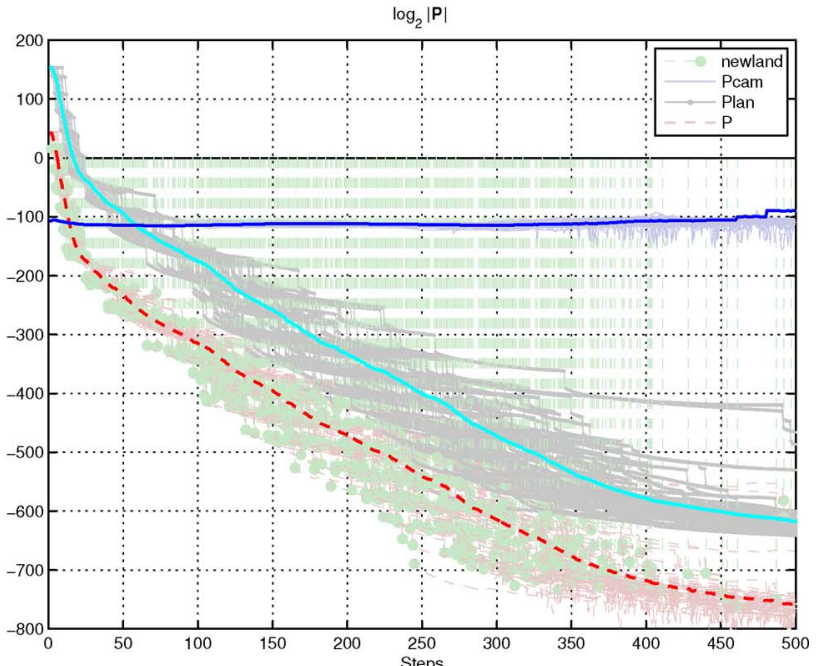

(b)

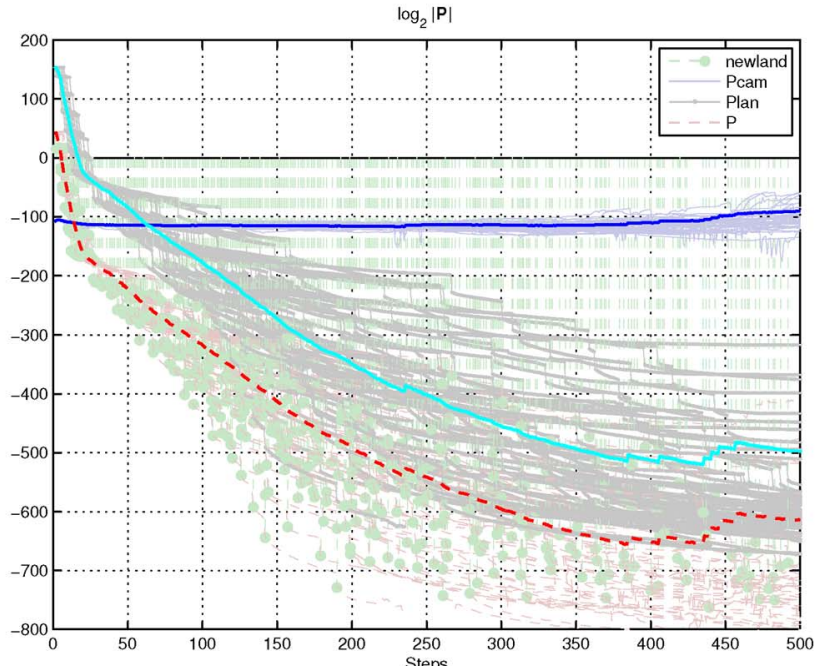

(d)

Fig. 4. Monte Carlo simulation of 100 runs for the freely moving single camera using an unconstrained motion model with two different action selection mechanisms: (a) and (b) mutual information and (c) and (d) random inputs. In (a) and (c), trajectories and a final map are plotted, and $r_{\text {Real }}$ and $r_{\text {Est }}$ are the real and estimated camera trajectories, respectively. In (b) and (d), Pcam, Plan, and P indicate the camera, map and overall entropies, respectively; the label newland and the green dots and dotted vertical lines represent the value of entropy at the instant when new landmarks are initialized. Mean values of the trajectories and the entropies are highlighted to ease comparison.

informative translation commands, letting the user freely choose where to look.

The set of possible actions is divided into seven elements, i.e., "go_forward," "go_backwards," "go_right," "go_left," "go_up," "go_down," and "stay." Details on the EKF-SLAM implementation are further detailed in [1].

1) Simulations: In our simulations of a freely moving camera, the desired camera pose is predicted for the best chosen action at each iteration. A feedback linearization low-level control law is applied to ensure that these locations are reached at 1-s intervals, at this point in time the motion metric is again evaluated to determine the next desired action.

The simulation considers a fixed number of expected landmarks to be found, and the mutual information metric is computed, taking into account such fixed number of landmarks in the computation of the innovation covariance. Unvisited landmarks are initialized with independently distributed large uncertainties of $1.5 \mathrm{~m}^{2}$.
A thorough evaluation of the proposed approach is presented with a 100-run Monte Carlo simulation. Parameters for the simulations are given in Table I.

Results of the Monte Carlo simulation are plotted in Figs. 4 and 5. Note the abrupt reductions in entropy in Fig. 4(b). These changes are caused by repeated small-loop closure events produced by autonomous back-and-forth or left-right maneuvers. This behavior is the product of the action selection mechanism chosen and is meant to keep the system well localized before attempting to search for new landmarks. Homoeostasis is avoided however through observation in the camera field of view of unvisited landmarks, whose corresponding actions produce large reductions in entropy and thus induce exploration.

The simulated environment represents a room that is $6 \times$ $6 \times 2.5 \mathrm{~m}^{3}$ in size containing 33 randomly distributed point landmarks, six of which are anchors, which are used as global references [30], [31]. These anchors make the system observable and provide a metric scale to the visual system. 


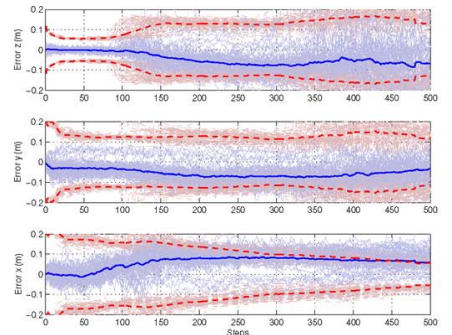

(a)
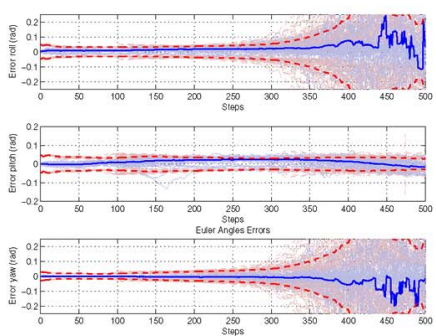

(b)

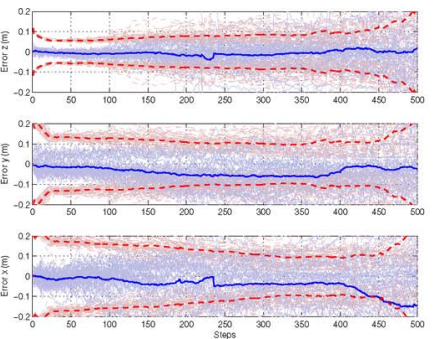

(c)

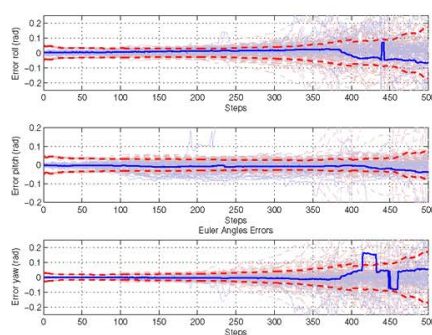

(d)

Fig. 5. Estimation errors for position and orientation of a 100-run Monte Carlo simulation for the freely moving single camera using an unconstrained motion model with two different action selection mechanisms: (a) and (b) mutual information action selection and (c) and (d) random input motion commands. Estimation errors for camera position and orientation, and their corresponding $2 \sigma$ standard deviation bounds are plotted. In (a) and (c), the position errors are indicated as distances to the real camera location along the $x$-, $y$-, and $z$-axis. In (b) and (d), orientation errors are given in Euler angles from the true orientation. Mean values are highlighted for comparison.

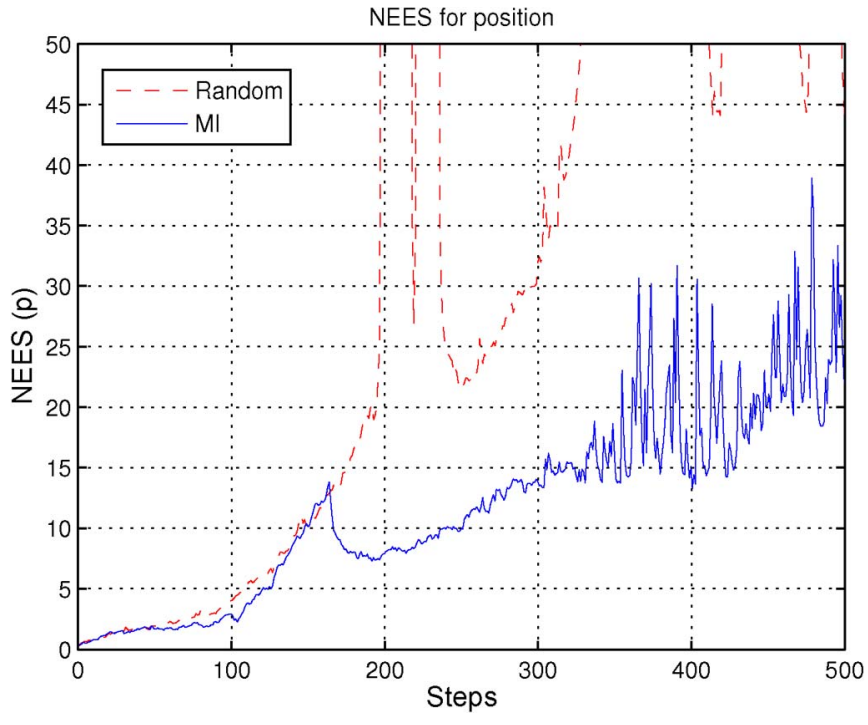

Fig. 6. Average NEES of the vehicle position p over 100 Monte Carlo runs. (Dotted red line) Random action selection strategy. (Continuous blue line) Average NEES for the mutual information action selection strategy.

Sensor standard deviation is set at 2 pixels, and data association is not known a priori. Instead, nearest neighbor $\chi^{2}$ tests are computed to guarantee correct matching. New landmarks are initialized once their ratio of depth estimate to depth standard deviation falls below a threshold of 0.3 . The plots show the results of actively translating a 6-DOF camera while building a map of 3-D landmarks. In all cases, each of the seven motion actions will produce a displacement of $0.3 \mathrm{~m}$ in the corresponding direction while rotating at a constant velocity of $0.1 \mathrm{rad} / \mathrm{s}$.

Figs. 4(c) and (d) and 5(c) and (d) show the results of controlling the camera with random inputs. Comparing these plots with our proposed strategy, we notice that, while our strategy reduces the entropy re-observing landmarks before searching for new landmarks, the random selection of actions is more eager to explore, without any control over loop closure and, hence, without worrying for localization accuracy. In the end, the result is improved entropy reduction for the proposed technique.

Improved localization of camera and landmarks is further shown through a normalized estimation error (NEES) plot of the mean Monte Carlo estimates, as explained in [32]. Fig. 6

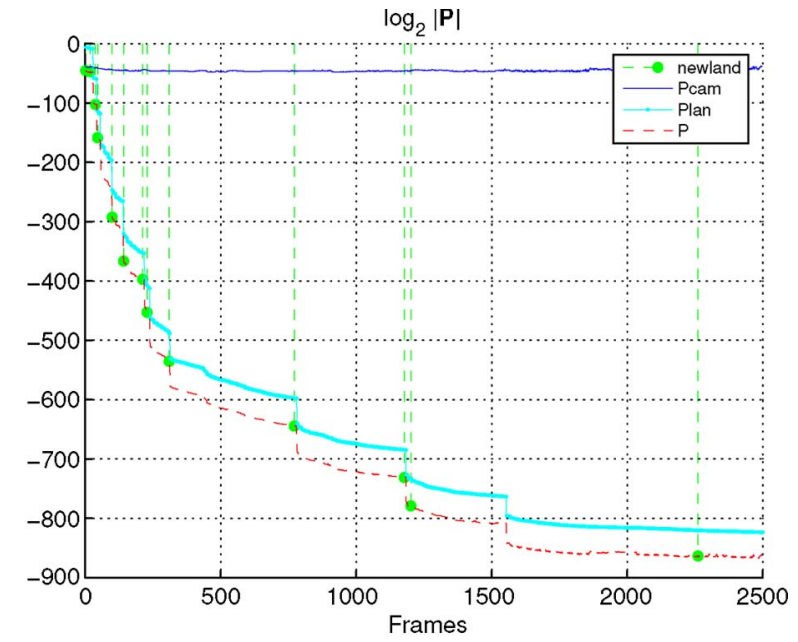

(a)

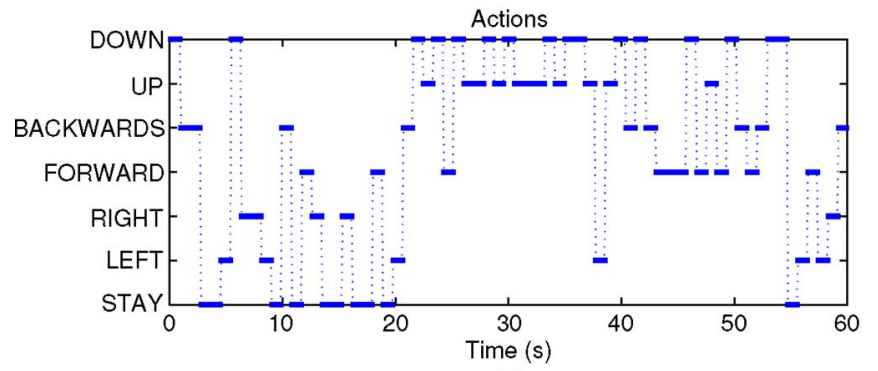

(b)

Fig. 7. Real-time active visual SLAM with a handheld camera experiment. (a) Computed camera, map, and total entropies during the experiment. The vertical lines indicate the points in time where new features are discovered. (b) List of actions sent to the GUI for the first minute.

shows that the average NEES for the proposed approach is significantly better than that of the random action selection, justifying the validity of using entropy measures for action selection.

2) Real-Time Experiments: We present now experimental results validating the mutual information maximization strategy for the control of a handheld camera in a challenging 15-fps visual SLAM application. The experiments were implemented on top of the single-camera SLAM [33]. We developed an extension for this application that computes the desired actions using the approach presented in this paper sending motion commands to the GUI. 

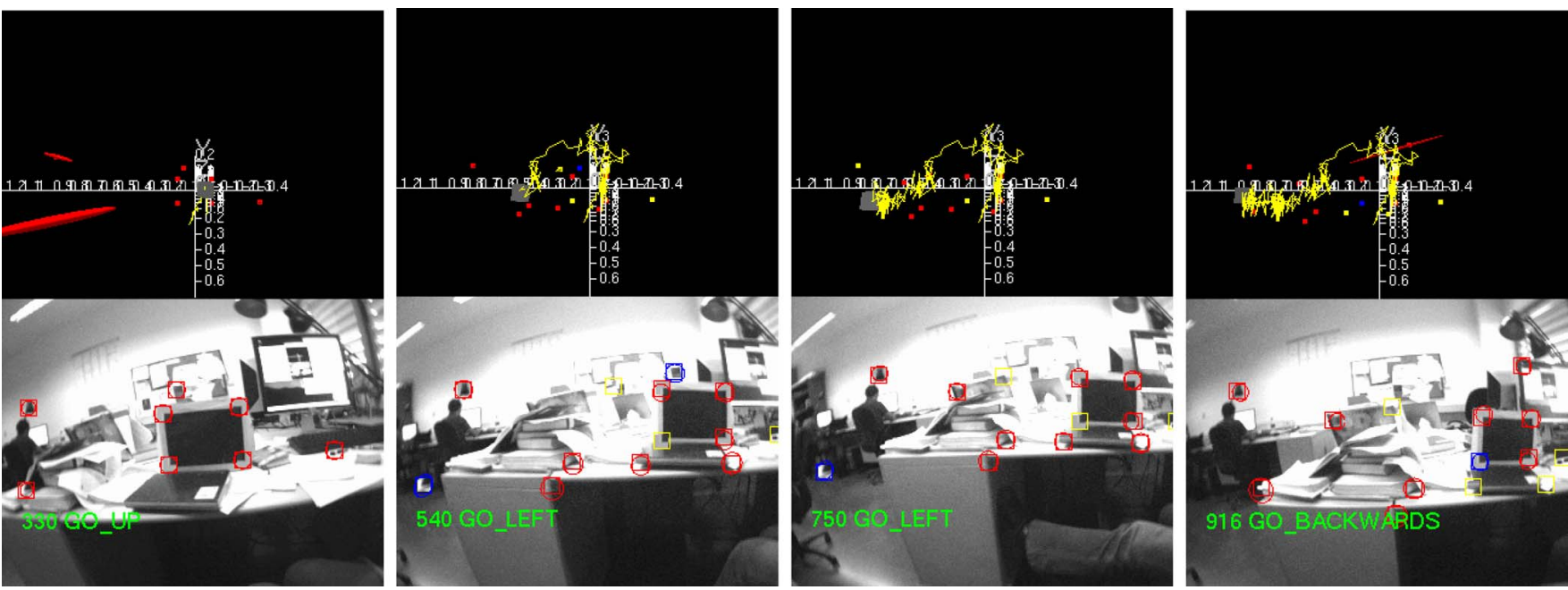

Fig. 8. Snapshots of the GUI during active visual SLAM experiment.

The camera starts approximately at rest with some known object in view to act as a starting point and provide a metric scale. The camera moves, translating and rotating in 3-D, according to the instructions provided to a user through a GUI and executed by the user, within a room of a restricted volume, such that various parts of the unknown environment come into view.

Given that the control loop is being closed by the human operator, only displacement commands are computed. Gaze control is left to the user. Furthermore, the mutual information measure requires evaluating the determinant of the innovation covariance matrix at each iteration. Because of the complexity of this operation, single-motion predictions are evaluated one frame at a time. It is not until the 15 th frame in the sequence that all mutual information measures are compared, and a desired action is displayed on screen. That is, the user is presented with motion directions to obey every second. Note also that, in computing the mutual information measure, only the camera position and map parts of the covariance matrix are used, leaving out the gaze and velocity parts of the matrix. Finally, to keep it running in real time, the resulting application must be designed for sparse mapping. That is, with the computing capabilities of an off-the-shelf system, our current application is limited to less than 50 landmarks.

Fig. 7 shows (a) a plot of the decrease in the various entropies for the map being built and (b) the list of actions chosen as shown to the user during the first minute. Note that, in consonance with our simulated exercise, in the realtime implementation, the system prompts the user for repeated up-down movements, as well as left-right commands. This can be explained as, after initializing new features, the system repeatedly asks for motions perpendicular to the line of sight to best reduce their uncertainty, to gain parallax. In addition, closing loops have an interesting effect in the reduction of entropy, as can be seen around the 1500th frame in Fig. 7(a).

Fig. 8 shows snapshots of the GUI for one of our validation experiments. The top part of each frame contains a 3-D plot of the camera and the landmarks mapped, whereas the bottom part shows the information being displayed to the user superimposed on the camera view.
TABLE II

ACtion Set For a Nonholonomic Vehicle

\begin{tabular}{|c|c|c|c|c|c|c|c|}
\hline Action & 0 & 1 & 2 & 3 & 4 & 5 & 6 \\
\hline Linear Acceleration & 0 & 0 & 0 & $-\dot{v}_{r}$ & $\dot{v}_{r}$ & $-\dot{v}_{r}$ & $\dot{v}_{r}$ \\
\hline Angular Acceleration & 0 & $-\dot{\omega}_{r}$ & $\dot{\omega}_{r}$ & 0 & 0 & $-\dot{\omega}_{r}$ & $\dot{\omega}_{r}$ \\
\hline
\end{tabular}

TABLE III

PARAMETERS FOR THE Simulation OF CONSTRAINED 3-D SLAM

\begin{tabular}{|c|c|}
\hline Initial pose & {$[0 \mathrm{~m}, 0 \mathrm{~m}, 0 \mathrm{~m}, 0 \mathrm{rad}, 0 \mathrm{rad}, 0 \mathrm{rad}]$} \\
\hline $\begin{array}{c}\text { Initial pose } \\
\text { variances }\end{array}$ & {$[1 \mathrm{~m}, 0.5 \mathrm{~m}, 1 \mathrm{~m}$,} \\
\hline Actions & $0.1 \mathrm{rad}, 0.1 \mathrm{rad}, 0.1 \mathrm{rad}]$ \\
& $\dot{v}_{r}=0.3 \mathrm{~m} / \mathrm{s}^{2}$ \\
& $\dot{\omega}_{r}=0.05 \mathrm{rad} / \mathrm{s}^{2}$ \\
\hline
\end{tabular}

\section{B. Vehicle in 3-D Environment}

Now we present the guidance of a vehicle performing SLAM with a single wide-angle camera in uneven terrain. Actions in the form of impulse accelerations guarantee smooth platform velocity change. The chosen command is then integrated to produce the input velocity that is sent to the vehicle. The actions are chosen from the discrete set from Table II.

To compare the effects produced by the various actions on the localization and map estimates, the motion model and a feedback control law are used to predict the prior mean $\mathbf{x}_{k+1 \mid k}$ for each instant acceleration in the set, propagating the covariances by computing the corresponding Jacobians. Map feature priors are also used to simulate the expected observations using the camera measurement model and the state prior. The posterior covariance is then computed, taking into account only known landmarks inside the camera field of view.

1) Simulations: A Monte Carlo simulation with 100 runs of a mobile robot navigating in uneven terrains is executed. Plant noise varied using the constrained motion model in (7), using the full-perspective wide-angle camera model and a sinusoidal model of a 3-D surface. In our simulations, as well as in the real-time experiments, encoder velocities are used to predict plane surface normals for the computation of action priors constrained to the characteristics of the terrain. The simulated environment contains 31 landmarks whose location is sampled 


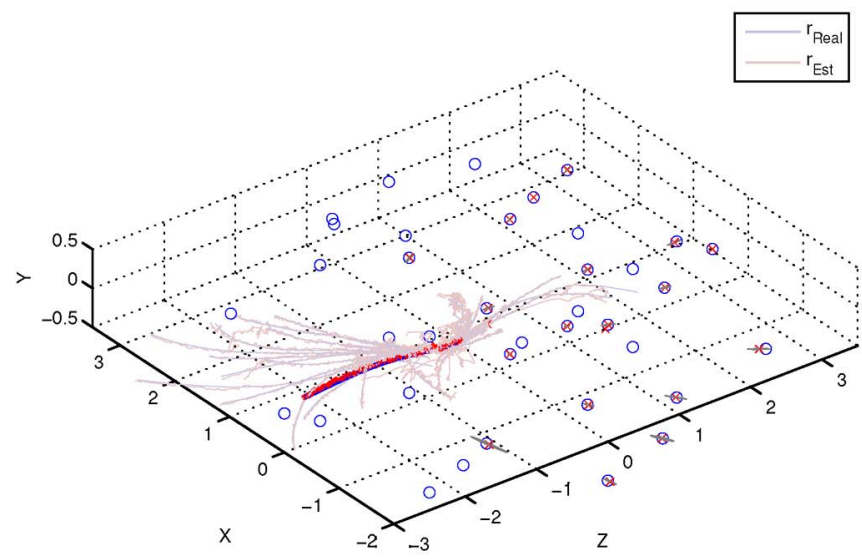

(a)

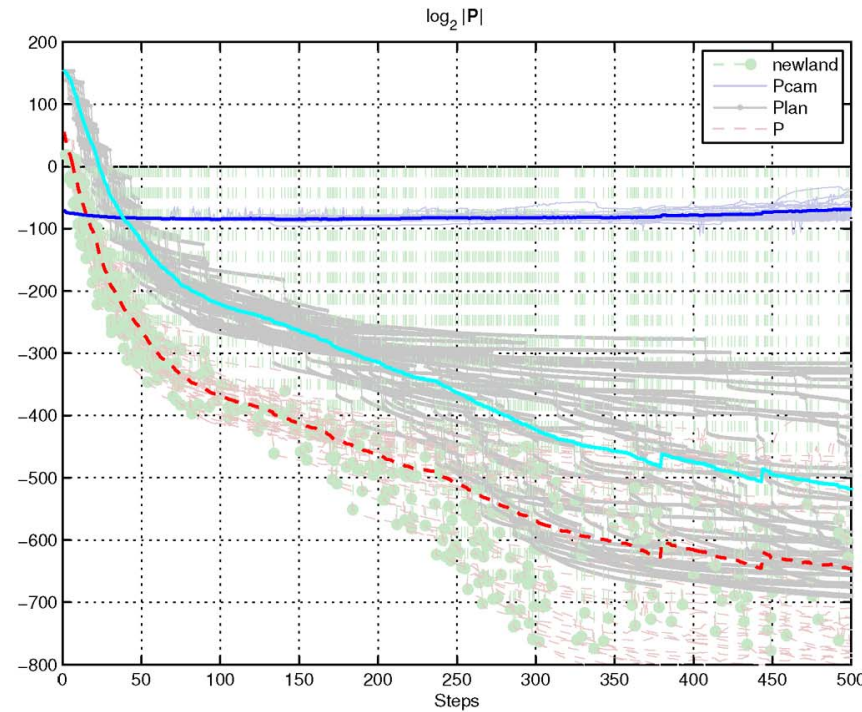

(b)

Fig. 9. Camera trajectories and entropies of a 100-run Monte Carlo simulation of a mobile robot exploring an uneven room. The mutual information maximization strategy produces a nearly linear motion tangent to the surface. The vehicle starts at the shown terrain depression and proceeds backward, slightly rotating to increase map coverage. In (a), labels $r_{R e a l}$ and $r_{E s t}$ indicate real and estimated vehicle trajectories, respectively. In (b), Pcam, Plan, and P indicate the robot, map, and overall entropies. In addition, in (b), the label newland and the green dots, and the dotted vertical lines represent the value of entropy at the instant when new landmarks are initialized.
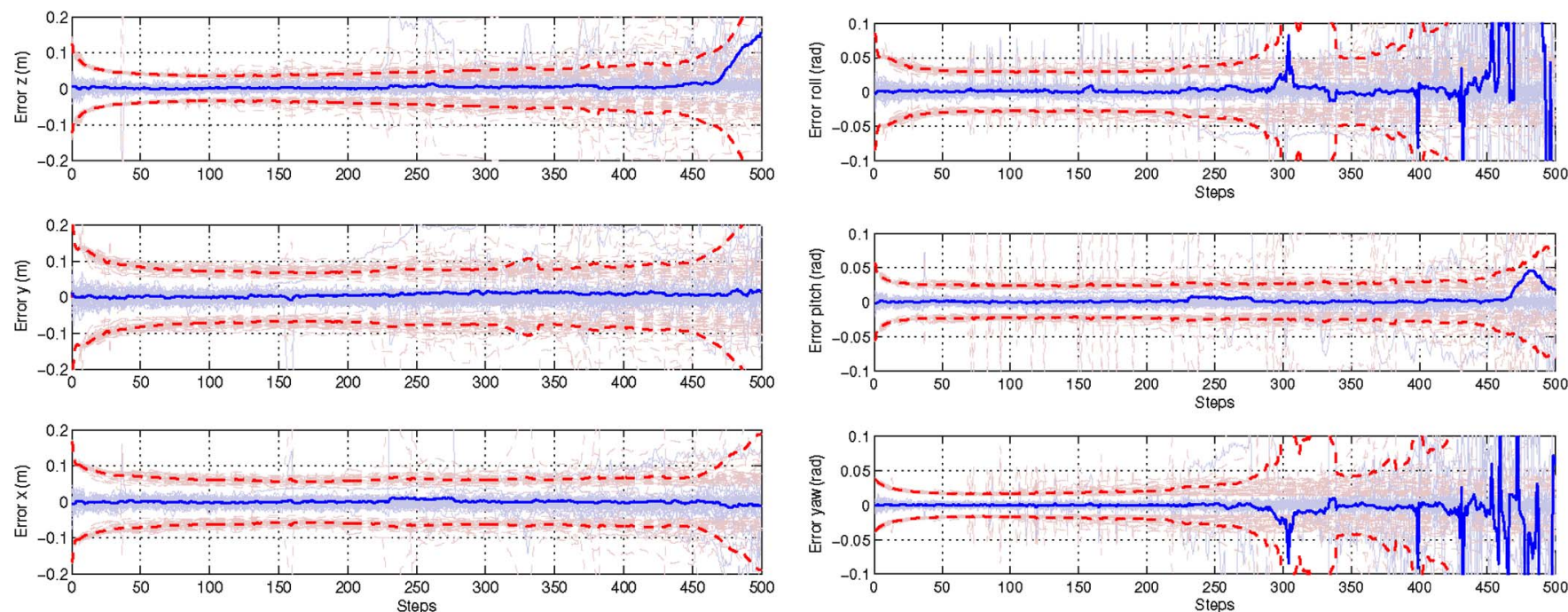

(a)

(b)

Fig. 10. (a) Estimation errors for camera position and (b) orientation and their corresponding $2 \sigma$ standard deviation bounds, respectively. In (a), position errors are plotted as distances to the real camera location in meters along the $x-, y$-, and $z$-axis. In (b), orientation errors are plotted in Euler angles.

from a uniform distribution. Of these, six are used as anchors to give a metric scale and help fulfil the observability conditions of the SLAM system. The rest is initialized with $5-\mathrm{m}^{2}$ variance to avoid homoeostasis. Other simulation parameters are given in Table III.

Fig. 9 shows the vehicle trajectories and the landmark locations with their uncertainty plotted using hyper-ellipsoids of uncertainty at $2 \sigma$. The steep decays in overall entropy indicate either landmark discovery or repeated loop closure. Interestingly enough, the nonholonomic motion constraints of the system force the vehicle to autonomously explore by repeatedly choosing a negative linear acceleration. The effect is to augment the camera field of view with the consequent inclusion of new features in the model but still maintaining known landmarks in sight, thus keeping the vehicle well localized at all times. In contrast to the unconstrained motion case, short-loop closures orthogonal to the field of view to maintain parallax are not possible to achieve, with the reason being that the vehicle cannot perform saccadic motions in the way that a free-moving camera can. Loop closure instead is only possible through backand-forth motion sequences.

Camera pose estimation errors are shown in Fig. 10. Notice how, when the terrain abruptly changes, velocities become underestimated in the direction that the terrain changed. Thus, 


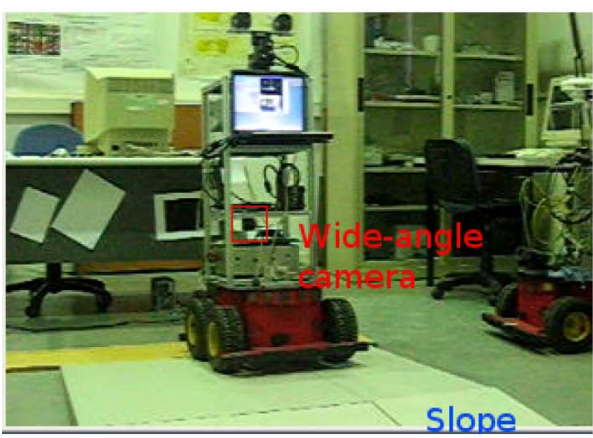

(a)
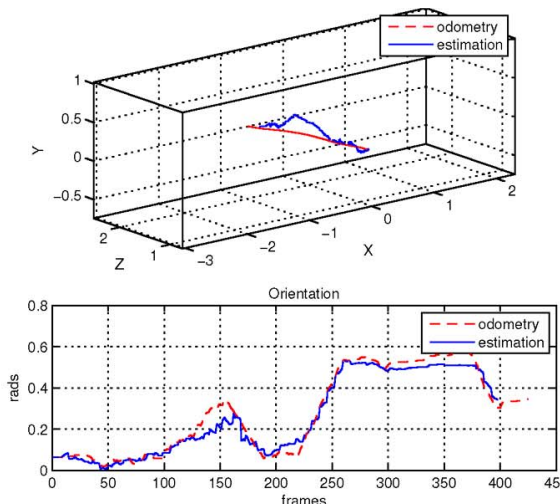

(b)

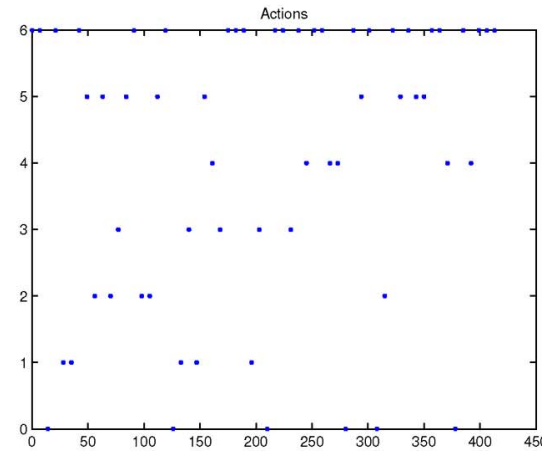

(c)

Fig. 11. Real-time experiment of a single camera mounted on a vehicle traversing uneven terrain. (a) Mobile robot used in the experiments. (b) 3-D trajectory of the vehicle and orientation with respect to the $y$ axis. (c) Actions from Table II sent to the vehicle every second.

in simulating vehicle motion, a more elaborate model taking into account surface discontinuities must be considered for very rough terrains.

2) Real-Time Experiments: For the case where the camera is mounted on a vehicle, two independent modules are considered: an action selection module on top of our modified version of the SLAM system [33] and an interface that communicates such motion commands to the robot, enforcing low-level obstacle avoidance and system integrity. The robot is controlled using ARIA [34], and the experiments were conducted on the mobile platform shown in Fig. 11, with a wide-angle camera rigidly attached to the robot body.

The whole process is running at 15 fps. Single-motion predictions are evaluated one frame at a time. It is only every seventh frame in the sequence that all mutual information measures are compared, and the best action is sent to the mobile robot. For the experiments, the acceleration magnitudes were set to $\dot{v}_{r}=0.1 \mathrm{~m} / \mathrm{s}^{2}$ and $\dot{\omega}_{r}=0.09 \mathrm{rad} / \mathrm{s}^{2}$. When computing posteriors, these are predicted for the duration that would take them to the end of the 15 th frame, with each action in turn being evaluated for a slightly shorter period of time. The motivation is that we want to be able to test actions on the basis of their effect at the very same point in time (at the end of the 15th frame). In order to evade any bias related to the time spent in evaluating the effect of actions, these are randomly ordered at each iteration.

As with the simulated setting, the robot navigates in uneven terrain, as shown in Fig. 11. In the plots, the estimated path (blue continuous line in top frame) is shown in 3-D, as opposed to the vehicle odometry, which is restricted to the $x z$ plane. The orientation angle (bottom frame) indicates the vehicle orientation with respect to the world axis $y$ (orthogonal to the white sheet of paper placed in front of the robot, which serves as global reference consistent to the world $x z$ plane).

As in the simulated case, our mutual information-based action selection strategy for this constrained motion case autonomously explores the room driving the vehicle back and forth but mostly backward, enlarging the field of view by pulling away from the initial view. Fig. 11(b) gives account of the actions sent to the robot and shows as most frequent action iterations between positive and negative linear accelerations.
The feature map and camera pose are updated and displayed in real time in the GUI. Fig. 12 contains a sequence of frames from the experiment that shows the robot driving away from the initial known features.

We have shown with our simulations and experiments how the principled action selection mechanism proposed can deal with both unconstrained and constrained motion models, automatically selecting the best suited actions for each case and enforcing minimization of localization and map estimation uncertainty during SLAM exploration sessions. For the unconstrained motion model, saccadic motion perpendicular to the line of sight is selected since it closes small loops as features are discovered, actively reducing entropy. For the nonholonomic constrained motion model, these saccadic movements are not possible, and the system settles for moving backward, increasing the field of view and adding new features to the stare estimate while preserving observation of previous ones, again actively enforcing loop closure and reduced estimation entropy.

\section{Obstacle Avoidance}

We consider now the case of longer term navigation, combining the goals of exploration and navigation to a specific location. In this case, low-level obstacle avoidance as the one implemented in the previous section does not suffice, and a higher level mechanism to help drive the robot through obstacles should be implemented. We presented in Section IV-C a control law that weighs these two objectives during robot navigation: 1) exploration and 2) obstacle avoidance.

The technique uses APFs to enforce smooth maneuvering through the obstacles that the SLAM algorithm discovers during exploration. We present the result of a Monte Carlo simulation with 100 runs, in which the robot is driven through a path of more than $3 \mathrm{~m}$ in length, with the goal location at $(0$, $0,2)$. The attraction potential function scaling factor is $\psi=1$, the repulsive potential factor is $\nu=2$, and the safe radio for obstacles is $\rho=0.35 \mathrm{~m}$. Other simulation parameters are given in Table IV.

The results of using the APF method without active entropybased action selection are shown in of Figs. 13(a) and (b) and 14(a) and (b). The plots highlight the mean trajectory and mean 

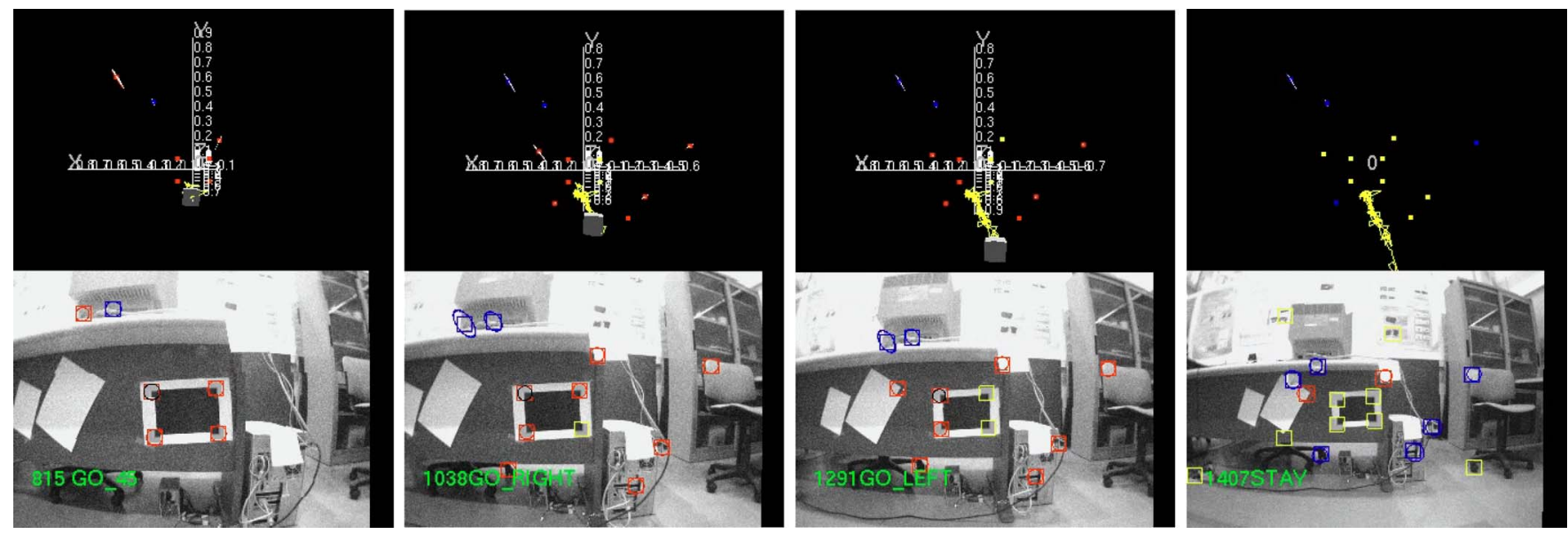

Fig. 12. Snapshots of the GUI during the autonomous exploration of a single camera mounted on a vehicle traversing uneven terrain.

TABLE IV

PARAMETERS For the Simulation OF CONSTRAined 3-D SLAM With ARTIFICIAL Potential FieldS

\begin{tabular}{|c|c|}
\hline $\begin{array}{l}\text { Initial } \\
\text { pose }\end{array}$ & $\begin{array}{c}{[0 \mathrm{~m}, 0 \mathrm{~m},-1.25 \mathrm{~m},} \\
0 \mathrm{rad}, 0 \mathrm{rad}, 0 \mathrm{rad}]\end{array}$ \\
\hline $\begin{array}{c}\text { Initial pose } \\
\text { variances }\end{array}$ & $\begin{array}{c}{[0.1 \mathrm{~m}, 0.05 \mathrm{~m}, 0.1 \mathrm{~m}} \\
0.05 \mathrm{rad}, 0.01 \mathrm{rad}, 0.01 \mathrm{rad}]\end{array}$ \\
\hline Actions & $\dot{v}_{r}=0.3 \mathrm{~m} / \mathrm{s}^{2}, \dot{\omega}_{r}=0.05 \mathrm{rad} / \mathrm{s}^{2}$ \\
\hline
\end{tabular}

entropy estimates for the entire Monte Carlo runs. The sequence of control inputs is given in Fig. 15.

The strategy is compared with our hybrid control law, which takes into account obstacle avoidance, as well as reduction of entropy. Figs. 13(c) and (d) and 14(c) and (d) show the trajectories, entropy plots, and position and orientation error plots. Note how obstacle collision is gracefully avoided, whereas the SLAM system effectively maximizes the mutual information.

The advantage of using the hybrid technique can be observed in the entropy plots in Fig. 13. The system spends more time gazing to already mapped landmarks, instead of driving the robot toward the goal, and adds landmarks to the map at later instants in time. The entire simulation takes 900 steps, on average, instead of the 700 steps that the pure APF system takes. Thus, the result is a compromise between execution time and accuracy in estimation results. Another advantage of the hybrid approach is that the mutual information term in the action law helps the system overcome local minima traps common in APF navigation.

\section{CONCLUSION}

Action selection strategies for guiding a video-rate visual SLAM system have been introduced and shown using maximal mutually informative motion commands by maximizing the difference between innovation covariance and posterior SLAM entropies. The resulting motion command reduces the uncertainty of the state from the information given by measurements.

Our method is validated in three visual SLAM implementations: 1) a video-rate handheld SLAM system; 2) a mobile robot with nonholonomic and terrain constraints; and 3) an
APF for obstacle avoidance. The video-rate handheld camera system produces motion commands in real time for 6-DOF visual SLAM and is sufficiently general to be incorporated into any type of mobile platform, without the need for other sensors. The action selection mechanism actively performs short-loop closure orthogonal to the field of view of the camera.

Mounting the same wide-angle camera on a wheeled mobile robot provides for an autonomous information-driven exploration strategy that has been tested for navigation in uneven terrains. Simulation and experimental results consistently show a behavior in which the robot pulls back from its initial configuration, having the camera search for more landmarks while reducing its own pose uncertainty. In the end, we have a simple principled action selection mechanism that seems to accommodate for unconstrained and constrained motion models, choosing the most appropriate actions in each case and guaranteeing reduced estimation uncertainty during exploration.

The reported camera trajectories are simple, because 1) the robot is commanded by acceleration impulses that tend to drive the robot through smooth velocity changes and 2) the real-time constraints of the implementation allow only for the evaluation of a very limited set of possible actions. The computational complexity in computing entropy does not permit large maps; in that case, submapping will be a good solution.

In a third experimental setting, our strategy is combined with an obstacle avoidance mechanism that uses APFs. Actions are now chosen with a compromise between navigation toward a goal by using attractive potentials, avoiding obstacles on the way using repulsive potentials, and enforcing reduced estimation uncertainty through entropy minimization.

The real-time requirements of the task preclude using an optimal control law that uses a continuous-valued action space. Instead, we evaluate our information metrics for a small set of actions carried out over a fixed amount of time and choose the best action from those. Improvements can be done with greedy algorithms, which consider the evaluation of $n$-step lookahead and also larger sets of actions. These improvements are issues of further study.

One possible weakness of the presented information-based approach to action selection is that it estimates the utility of measures, assuming correct motion and measurement models. 


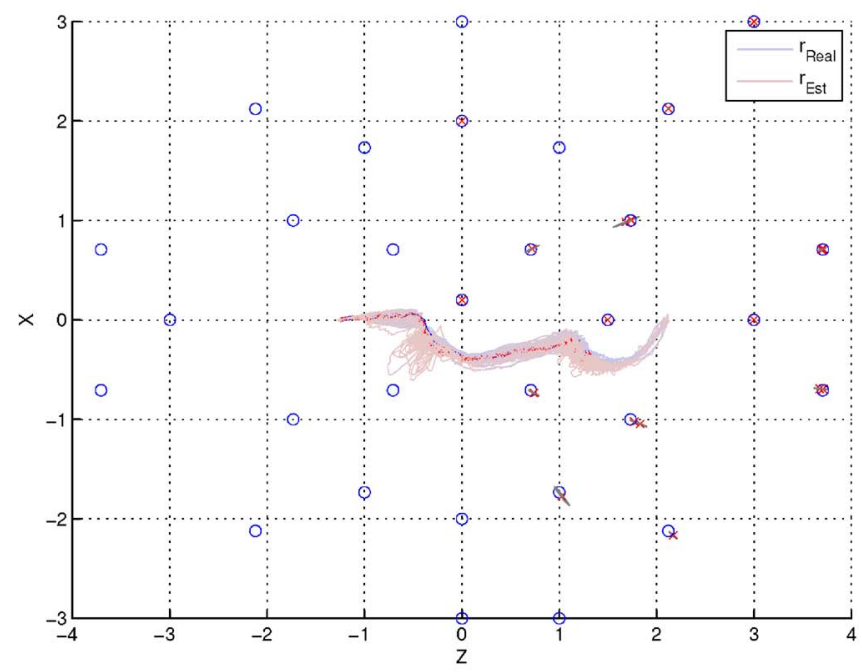

(a)

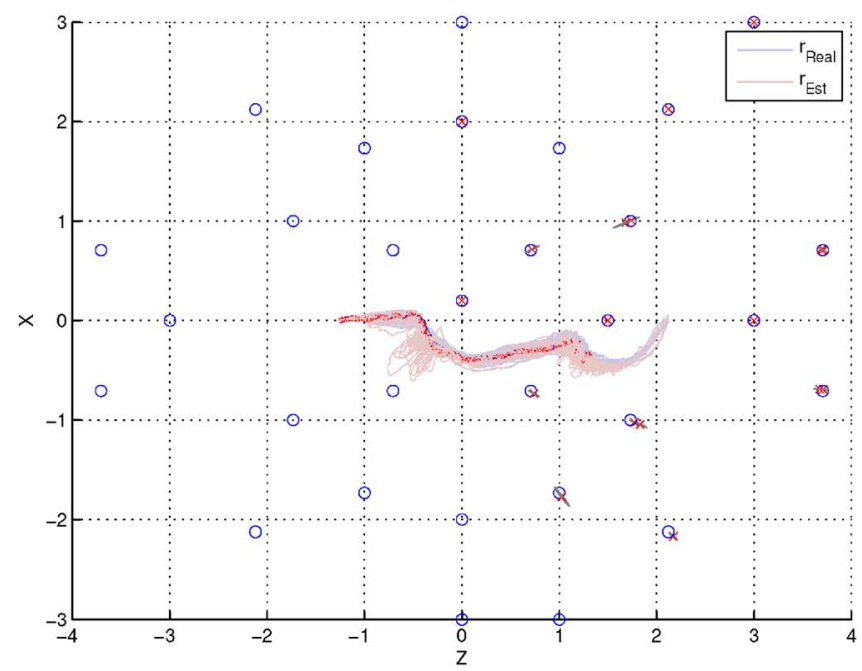

(c)

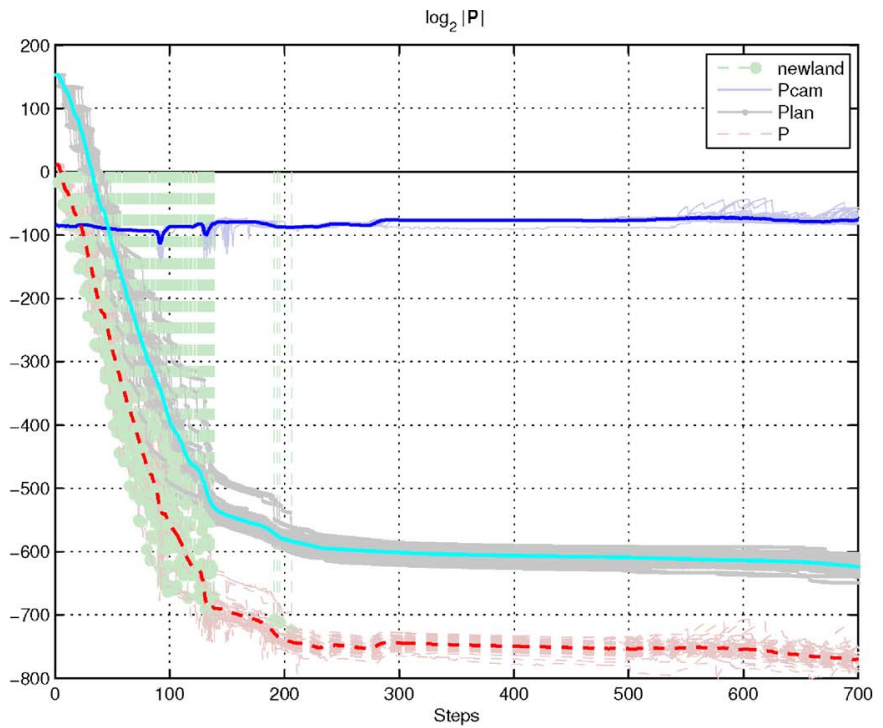

(b)

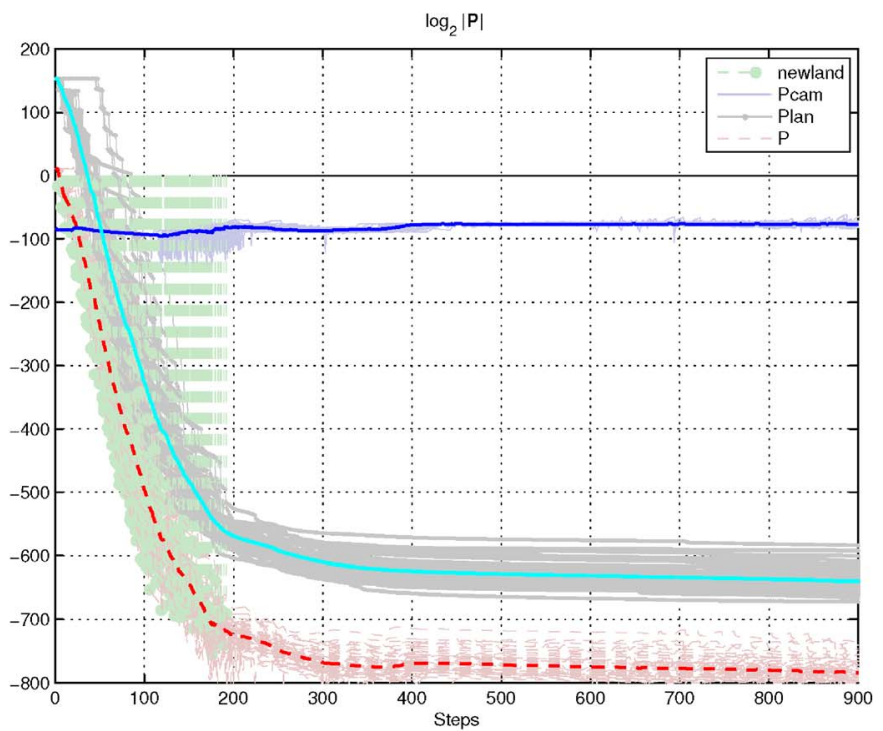

(d)

Fig. 13. (a) and (c) Vehicle trajectories and map and (b) and (d) entropies of a 100-run Monte Carlo simulation of a mobile robot moving to the goal using (a) and (b) the APF strategy to control the vehicle and (c) and (d) APF combined with mutual information to control the vehicle. Map features are considered as obstacles. In (a) and (c), $r_{\text {Real }}$ and $r_{\text {Est }}$ indicate real and estimated vehicle trajectories, respectively. In (b) and (d), the label newland and the green dots and dotted vertical lines represent the value of entropy at the instant when new landmarks are initialized. Pcam and P indicate the robot, map, and overall entropies. The mean value of entropies is highlighted for comparison.

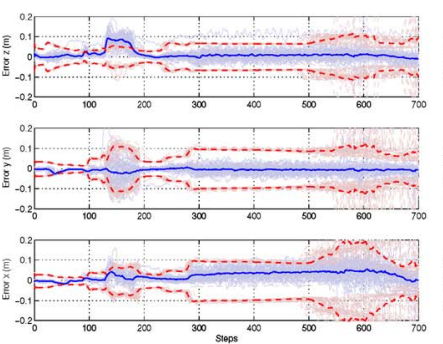

(a)

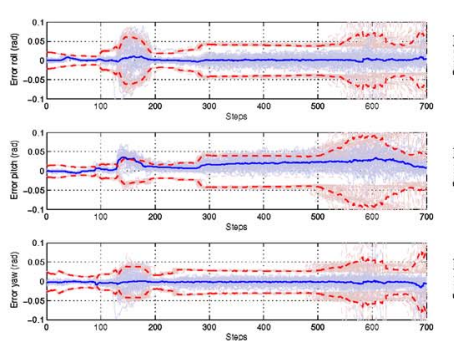

(b)

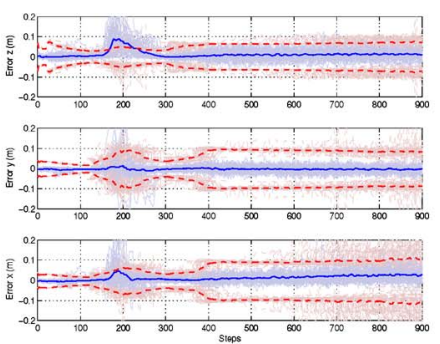

(c)

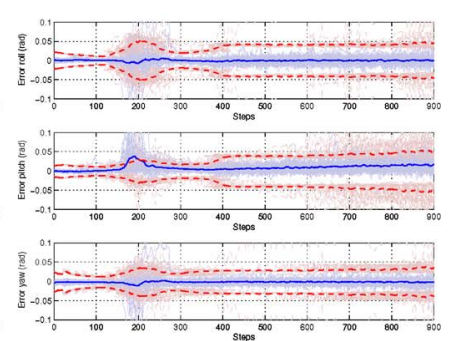

(d)

Fig. 14. (a) and (c) Camera position and (b) and (d) orientation errors for a Monte Carlo simulation with 100 runs. Standard deviation bounds are plotted at $2 \sigma$ for the APF strategy alone [(a) and (b)], and the hybrid method with APF and mutual information [(c) and (d)]. Position errors are plotted as $x, y$, and $z$ distances to the real camera location in meters, and orientation errors are plotted as Euler angles. The mean values are highlighted for comparison. 


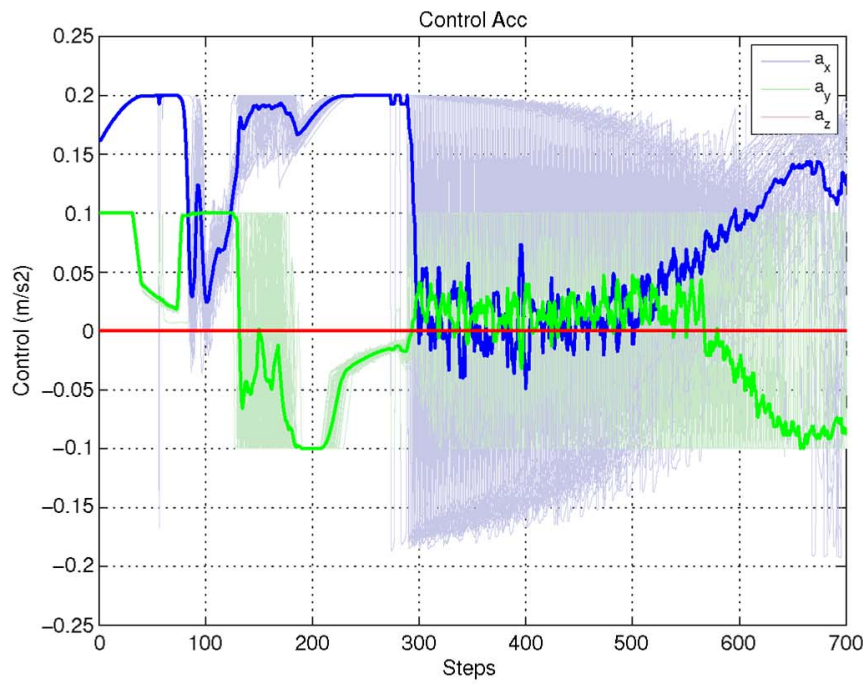

(a)

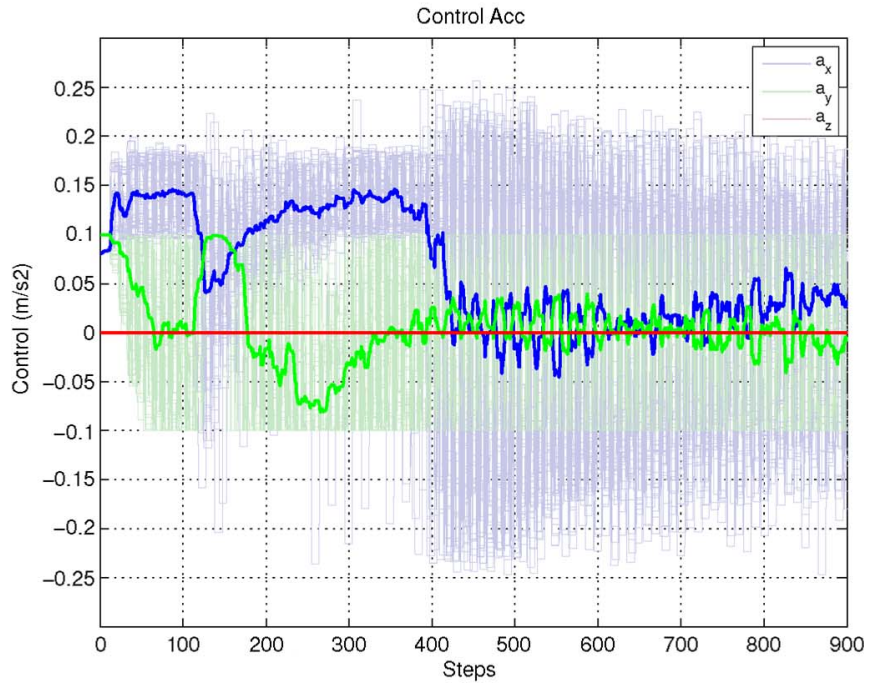

(b)

Fig. 15. Control command signals for the vehicle. (a) Accelerations produced by APF alone. (b) Accelerations produced by the combined APF with mutual information. The desired linear velocities are set to $0.2 \mathrm{~m} / \mathrm{s}$ in $x$ and $0.1 \mathrm{~m} / \mathrm{s}$ in $y$.

Model discrepancies and effects of linearization in the computation of estimates and control commands are not being considered and could play a major role for large sampling intervals. This issue is also a topic of further research.

\section{ACKNOWLEDGMENT}

The authors would like to thank A. Davison and D. Murray for the discussions and software collaboration.

\section{REFERENCES}

[1] A. Davison, "Real-time simultaneous local ization and mapping with a single camera," in Proc. IEEE Int. Conf. Comput. Vis., Nice, Oct. 2003, pp. 1403-1410.

[2] T. Vidal-Calleja, A. Davison, J. Andrade-Cetto, and D. Murray, "Active control for single camera SLAM," in Proc. IEEE Int. Conf. Robot. Autom., Orlando, FL, May 2006, pp. 1930-1936.
[3] T. Vidal-Calleja, A. Sanfeliu, and J. Andrade-Cetto, "Guiding and localising in real-time a mobile robot with a monocular camera in nonflat terrains," in Proc. 6th IFAC/EURON Symp. Intell. Auton. Vehicles, Toulouse, France, Sep. 2007, pp. 1-6.

[4] B. Kim, D. Roh, J. Lee, M. Lee, K. Son, M. Lee, J. Choi, and S. Han, "Localization of a mobile robot using images of a moving target," in Proc. IEEE Int. Conf. Robot. Autom., Seoul, Korea, May 2001, pp. 253-258.

[5] A. K. Das, R. Fierro, V. Kumar, B. Southhall, J. Spletzer, and C. J. Taylor, "Real-time vision-based control of a nonholonomic mobile robot," in Proc. IEEE Int. Conf. Robot. Autom., Seoul, Korea, May 2001, pp. 1714-1719.

[6] K. Usher, P. Corke, and P. Ridley, "Home alone: Mobile robot visual servoing," in Proc. IEEE/RSJ IROS Workshop Vis. Servoing, Lausanne, Switzerland, Oct. 2002, pp. 10-20.

[7] D. Xu, Y. Li, M. Tan, and Y. Shen, "A new active visual system for humanoid robots," IEEE Trans. Syst., Man, Cybern. B, Cybern., vol. 38, no. 2 , pp. 320-330, Apr. 2008.

[8] K. T. Song and J. H. Huang, "Fast optical flow estimation and its application to real-time obstacle avoidance," in Proc. IEEE Int. Conf. Robot. Autom., Seoul, Korea, May 2001, pp. 2891-2896.

[9] H. Y. Wang, S. Itani, T. Fukao, and N. Adachi, "Image-based visual adaptive tracking control of nonholonomic mobile robots," in Proc. IEEE/RSJ Int. Conf. Intell. Robots Syst., Maui, HI, Nov. 2001, pp. 1-6.

[10] J. Schmüdderich, V. Willert, J. Eggert, S. Rebhan, and C. Goerick, "Estimating object proper motion using optical flow, kinematics, and depth information," IEEE Trans. Syst., Man, Cybern. B, Cybern., vol. 38, no. 4, pp. 1139-1151, Aug. 2008.

[11] A. J. Davison and D. W. Murray, "Simultaneous localisation and mapbuilding using active vision," IEEE Trans. Pattern Anal. Mach. Intell., vol. 24, no. 7, pp. 865-880, Jul. 2002.

[12] P. Whaite and F. Ferrie, "Autonomous exploration: Driven by uncertainty," in Proc. 9th IEEE Conf. Comput. Vis. Pattern Recog., Seattle, WA, Jun. 1994, pp. 339-346.

[13] H. J. S. Feder, J. J. Leonard, and C. M. Smith, "Adaptive mobile robot navigation and mapping," Int. J. Robot. Res., vol. 18, no. 7, pp. 650-668, 1999.

[14] F. Bourgault, A. Makarenko, S. Williams, B. Grocholsky, and H. Durrant-Whyte, "Information based adaptative robotic exploration," in Proc. IEEE/RSJ Int. Conf. Intell. Robots Syst., Lausanne, Switzerland, Oct. 2002, pp. 540-545.

[15] C. Stachniss, G. Grisetti, and W. Burgard, "Information gain-based exploration using Rao-Blackwellized particle filters," in Proc. Robot.: Sci. Syst. I, Cambridge, MA, Jun. 2005, pp. 65-72.

[16] J. Porta, M. Spaan, and N. Vlassis, "Robot planning in partially observable continuous domains," in Proc. Robot.: Sci. Syst. I, Cambridge, MA, Jun. 2005, pp. 217-224.

[17] M. Bryson and S. Sukkarieh, "An information-theoretic approach to autonomous navigation and guidance of an uninhabited aerial vehicle in unknown environments," in Proc. IEEE/RSJ Int. Conf. Intell. Robots Syst., Edmonton, AB, Canada, Aug. 2005, pp. 3770-3775.

[18] J. Andrade-Cetto and A. Sanfeliu, Environment Learning for Indoor Mobile Robots. A Stochastic State Estimation Approach to Simultaneous Localization and Map Building. New York: Springer-Verlag, 2006, ser. Springer Tracts in Advanced Robotics.

[19] D. J. C. MacKay, "Information based objective functions for active data selection," Neural Comput., vol. 4, no. 4, pp. 589-603, Jul. 1992.

[20] R. Sim, "Stable exploration for bearings-only SLAM," in Proc. IEEE Int. Conf. Robot. Autom., Barcelona, Spain, Apr. 2005, pp. 2422-2427.

[21] C. Leung, S. Huang, N. Kwok, and G. Dissanayake, "Planning under uncertainty using model predictive control for information gathering," Robot. Auton. Syst., vol. 54, no. 11, pp. 898-910, Nov. 2006.

[22] R. Martinez-Cantin, N. de Freitas, A. Doucet, and J. Castellanos, "Active policy learning for robot planning and exploration under uncertainty," in Proc. Robotics: Sci. Syst. III, Atlanta, GA, Jun. 2007, pp. 334-341.

[23] V. Ila, J. M. Porta, and J. Andrade-Cetto, "Information-based compact Pose SLAM," IEEE Trans. Robot., vol. 26, no. 1, pp. 78-93, Feb. 2010.

[24] A. Adam, E. Rivlin, and I. Shimshoni, "Computing the sensory uncertainty field of a vision-based localization sensor," IEEE Trans. Robot. Autom., vol. 17, no. 3, pp. 258-267, Jun. 2001

[25] T. Broida, S. Chandrashekhar, and R. Chellappa, "Recursive 3-D motion estimation from a monocular image sequence," IEEE Trans. Aerosp. Electron. Syst., vol. 26, no. 4, pp. 639-656, Jul. 1990.

[26] O. Khatib, "Real time obstacle avoidance for manipulators and mobile robots," Int. J. Robot. Res., vol. 5, no. 1, pp. 90-98, 1986.

[27] J. Borenstein and Y. Koren, "The vector field histogram fast obstacle avoidance for mobile robots," IEEE Trans. Robot. Autom., vol. 7, no. 3, pp. 278-288, Jun. 1991. 
[28] S. S. Ge and Y. J. Cui, "New potential functions for a mobile robot path planning," IEEE Trans. Robot. Autom., vol. 16, no. 5, pp. 615-620, Oct. 2000

[29] A. Davison, W. Mayol, and D. Murray, "Real-time localisation and mapping with wearable active vision," in Proc. IEEE Int. Symp. Mixed Augmented Reality, Tokyo, Japan, Oct. 2003, pp. 315-316.

[30] J. Andrade-Cetto and A. Sanfeliu, "The effects of partial observability when building fully correlated maps," IEEE Trans. Robot., vol. 21, no. 4, pp. 771-777, Aug. 2005.

[31] T. Vidal-Calleja, M. Bryson, S. Sukkarieh, A. Sanfeliu, and J. AndradeCetto, "On the observability of bearing only SLAM," in Proc. IEEE Int. Conf. Robot. Autom., Rome, Italy, Apr. 2007, pp. 4114-4118.

[32] T. Bailey, J. Nieto, J. Guivant, M. Stevens, and E. Nebot, "Consistency of the EKF-SLAM algorithm," in Proc. IEEE/RSJ Int. Conf. Intell. Robots Syst., Beijing, China, Oct. 2006, pp. 3562-3568.

[33] A. Davison, Y. Gonzalez-Cid, and N. Kita, "Real-time 3D SLAM with wide-angle vision," in Proc. 5th IFAC/EURON Sym. Intell. Auton. Vehicles, Lisbon, Portugal, Jul. 2004.

[34] MobileRobots Inc., "Aria: Advanced Robotics Interface for Applications," Amherst, NH, 2006. [Online]. Available: http://www.activrobots.com/ software/aria.html

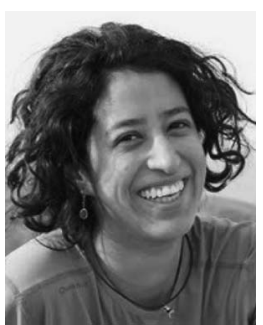

Teresa A. Vidal-Calleja received the M.E. degree from the Universidad Nacional Autónoma de México (UNAM), Mexico City, Mexico, the M.S.E.E. degree from Centro de Investigación y de Estudios Avanzados del Instituto Politécnico Nacional (CINVESTAV-IPN), Mexico City, and the $\mathrm{Ph} . \mathrm{D}$. degree from Universitat Politéctica de Catalunya (UPC), Barcelona, Spain, in 2007.

During her Ph.D. studies, she was Visiting Scholar with the Active Vision Lab, University of Oxford, Oxford, U.K., and the Australian Centre for Field Robotics (ACFR), University of Sydney, Sydney, NSW, Australia. In 2008, she was a Postdoctoral Fellow with LAAS-CNRS, Toulouse, France. She is currently on leave at the ACFR and is with the Institut de Robòtica i Informàtica Industrial, CSIC-UPC, Barcelona. Her current research interests include visual SLAM, cooperative aerial and ground vehicles, active sensing, and navigation.

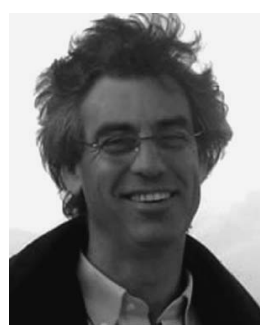

Alberto Sanfeliu received the B.S.E.E. and Ph.D. degrees from the Universitat Politéctica de Catalunya (UPC), Barcelona, Spain, in 1978 and 1982, respectively.

In 1981, he joined the faculty of UPC and is currently a Full Professor of computational sciences and artificial intelligence. He is also the Director of the Institut de Robòtica i Informàtica Industrial, CSIC-UPC, Barcelona. He was the Coordinator of the European-Union-funded URUS Project. He has authored books in pattern recognition and SLAM, and published more than 200 papers in international journals and conference proceedings. He is the holder of several patents on quality control based on computer vision. He works on various theoretical aspects on pattern recognition, computer vision, and robotics and on applications on vision defect detection, tracking, object recognition, robot vision, and SLAM.

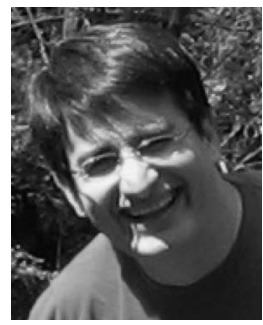

Juan Andrade-Cetto (S'94-M'95) received the B.S.E.E. degree from CETYS University, Mexico City, Mexico, in 1993, the M.S.E.E. degree from Purdue University, West Lafayette, IN, in 1995, and the Ph.D. degree in systems engineering from the Universitat Politéctica de Catalunya (UPC), Barcelona, Spain, in 2003.

$\mathrm{He}$ is currently an Associate Researcher of the Spanish National Research Council, Institut de Robòtica i Informàtica Industrial, CSIC-UPC, Barcelona, Spain. His current research interests include state estimation and computer vision with applications to mobile robotics. 